\title{
46. CLIMATICALLY INDUCED CHANGES IN VERTICAL WATER MASS STRUCTURE OF THE VEMA CHANNEL DURING THE PLIOCENE: EVIDENCE FROM DEEP SEA DRILLING PROJECT HOLES 516A, 517, AND 5181
}

\author{
David A. Hodell and James P. Kennett, Graduate School of Oceanography, University of Rhode Island, \\ Narragansett, Rhode Island \\ and \\ Kathleen A. Leonard, ${ }^{2}$ Department of Geology, University of South Carolina, Columbia, South Carolina
}

\begin{abstract}
Pliocene changes in the vertical water mass structure of the western South Atlantic are inferred from changes in benthic foraminiferal assemblages and stable isotopes from DSDP Holes 516A, 517, and 518. Factor analysis of 34 samples from Site 518 reveals three distinct benthic foraminiferal assemblages that have been associated with specific subsurface water masses in the modern ocean. These include a Nuttalides umbonifera assemblage (Factor 1) associated with Antarctic Bottom Water (AABW), a Globocassidulina subglobosa-Uvigerina peregrina assemblage (Factor 2) associated with Circumpolar Deep Water (CPDW), and an Oridorsalis umbonatus-Epistominella exigua assemblage associated with North Atlantic Deep Water (NADW). Bathymetric gradients in $\Delta^{13} \mathrm{C}$ between Holes 516A (1313 m), $517(2963 \mathrm{~m})$, and $518(3944 \mathrm{~m})$ are calculated whenever possible to monitor the degree of similarity and/or difference in the apparent oxygen utilization (AOU) of water masses located at these depths during the Pliocene. Changes in bathymetric $\Delta^{13} \mathrm{C}$ gradients coupled with benthic foraminiferal assemblages record fundamental changes in the vertical water mass structure of the Vema Channel during the Pliocene from 4.1 to $2.7 \mathrm{Ma}$.

At Site 518, the interval from 4.1 to $3.6 \mathrm{Ma}$ is dominated by the $N$. umbonifera (Factor 1 ) and $O$. umbonatus-E. exigua (Factor 3) assemblages. The $\Delta^{13} \mathrm{C}$ gradient between Holes $518(3944 \mathrm{~m})$ and $516 \mathrm{~A}(1313 \mathrm{~m})$ undergoes rapid oscillations during this interval though no permanent increase in the gradient is observed. However, $\delta^{13} \mathrm{C}$ values at Site 518 are clearly lighter during this interval. These conditions may be related to increased bottom water activity associated with the re-establishment of the West Antarctic Ice Sheet in the late Gilbert Chron ( 4.2 to 3.6 Ma) (Osborn et al., 1982).

The interval from 3.6 to $3.2 \mathrm{Ma}$ is marked by a dominance of the $G$. subglobosa-U. peregrina (Factor 2) assemblage and lack of a strong $\Delta^{13} \mathrm{C}$ gradient between Holes $518(3944 \mathrm{~m})$ and $516 \mathrm{~A}(1313 \mathrm{~m})$. We suggest that shallow circumpolar waters expanded to depths of a least $3944 \mathrm{~m}$ (Site 518) during this time.

The most profound faunal and isotopic change occurs at 3.2 Ma, and is marked by dominance of the $N$. umbonifera (Factor 1) and O. umbonatus-E. exigua (Factor 3) assemblages, a $1.1 \%$ enrichment in $\delta^{18} \mathrm{O}$, and a large negative increase in the $\Delta^{13} \mathrm{C}$ gradient between Holes 518 and 516A. These changes at Site 518 record the vertical displacement of circumpolar waters by AABW and NADW. This change in vertical water mass structure at $3.2 \mathrm{Ma}$ was probably related to a global cooling event and/or final closure of the Central American seaway. A comparison of the present-day $\delta^{13} \mathrm{C}$ structure of the Vema Channel with a reconstruction between 3.2 and $2.7 \mathrm{Ma}$ indicates that circulation patterns during this late Pliocene interval were similar to those of the modern western South Atlantic.
\end{abstract}

\section{INTRODUCTION}

Leg 72 of the Deep Sea Drilling Project (DSDP) has cored three sites along a depth transect down the Rio Grande Rise (Fig. 1). Each site intersects one of the major subsurface water masses of the western South Atlantic (Table 1). Leonard and others (this volume) report on the Pliocene paleoceanography of Hole 516A which records the history of changes in the position of Antarctic Intermediate Water (AAIW) and upper Circumpolar Deep Water (CPDW). Our study focuses upon the deeper water masses associated with Sites 517 and 518 . Site $517(2963 \mathrm{~m})$ is presently positioned in the core of North Atlantic Deep Water (NADW), and Site 518 (3944 m) is situated near large physical and chemical gradients separating Antarctic Bottom Water (AABW), lower Cir-

\footnotetext{
${ }^{1}$ Barker, P. F., Carlson, R. L., Johnson, D. A., et al., Init. Repts. DSDP, 72: Washington (U.S. Govt. Printing Office).

2 Present address: Marathon Oil Company, P.O. Box 3128, Houston, TX 77001.
}

cumpolar Deep Water (CPDW), and North Atlantic Deep Water (NADW) (Fig. 2). We have combined faunal and isotopic data from Holes 516A, 517, and 518 to potentially monitor changes in the vertical water mass structure of the Vema Channel during the Pliocene from 4.1 Ma to $2.7 \mathrm{Ma}$.

We are especially interested in determining the response of subsurface water masses to times of rapid climatic change. The climatic history of the Pliocene is marked by a high degree of stability punctuated by rapid climatic deterioration associated with the late Pliocene paleoceanographic events. The late Pliocene, at about 3.2 and $2.5 \mathrm{Ma}$, represents a time of conspicuous global cooling (Kennett et al., 1979; Keigwin and Thunell, 1979; Thunell, 1979), and the latter event (2.5 Ma) has been inferred to represent the beginning of substantial ice buildup on northern hemisphere continents (Shackleton and Opdyke, 1977). The purpose of this study is to investigate relationships between vertical migrations of water masses in the Vema Channel and times of climatic change during the Pliocene. 


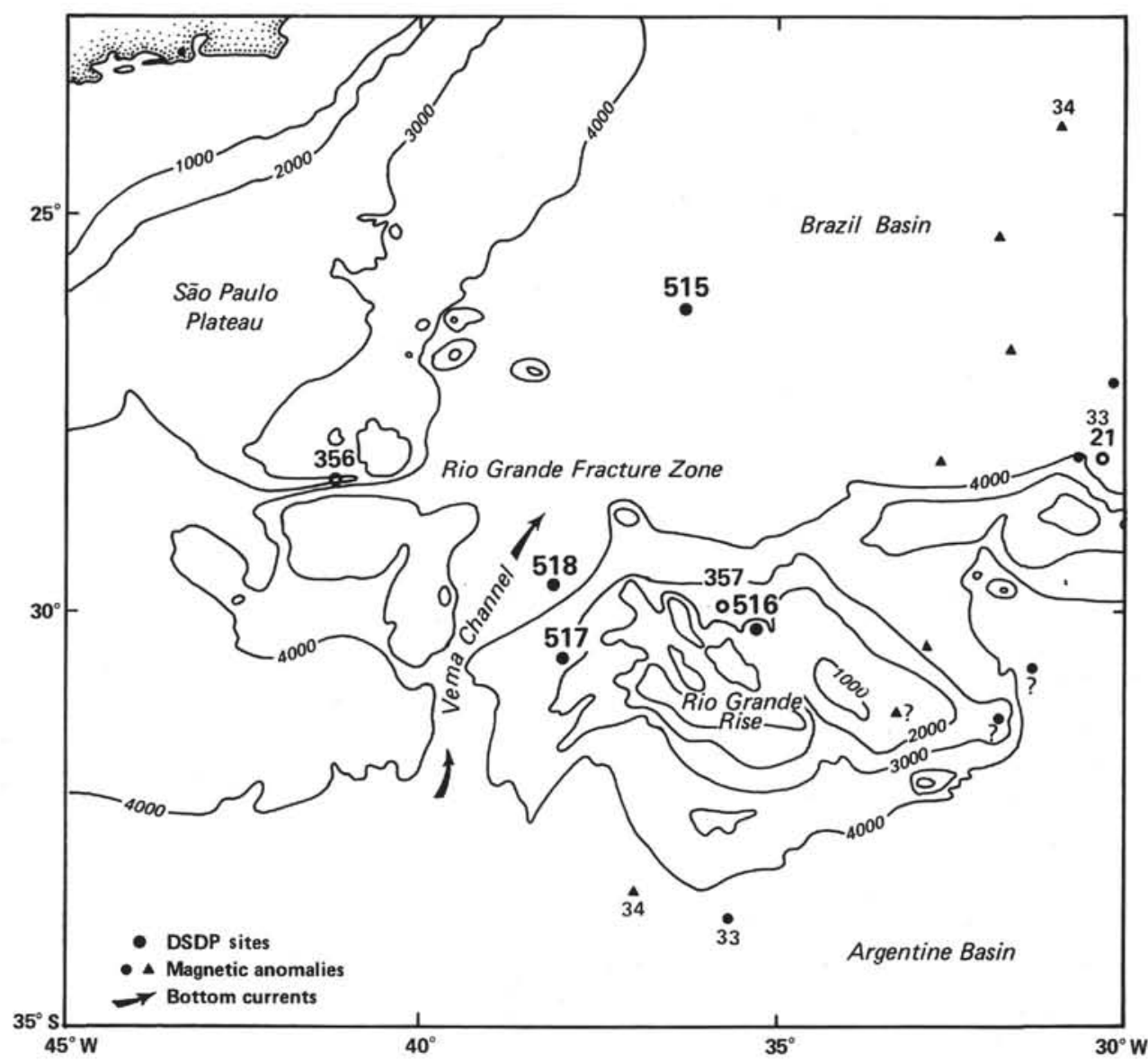

Figure 1 . The location of DSDP Holes 516A, 517, and 518, which were hydraulically piston cored along a depth transect down the Rio Grande Rise during Leg 72 in the southwestern Atlantic.

Table 1. Association of Leg 72 sites with subsurface water masses of the western South Atlantic.

\begin{tabular}{lcccl}
\hline Hole & Latitude & Longitude & $\begin{array}{c}\text { Water } \\
\text { depth } \\
(\mathrm{m})\end{array}$ & \multicolumn{1}{c}{ Water mass } \\
\hline 515 & $26^{\circ} 14.3^{\prime} \mathrm{S}$ & $36^{\circ} 30.2^{\prime} \mathrm{W}$ & 4250 & AABW \\
518 & $29^{\circ} 58.4^{\prime} \mathrm{S}$ & $38^{\circ} 08.1^{\prime} \mathrm{W}$ & 3944 & AABW, NADW, CPDW \\
517 & $30^{\circ} 56.8^{\prime} \mathrm{S}$ & $38^{\circ} 02.5^{\prime} \mathrm{W}$ & 2963 & NADW \\
$516 \mathrm{~A}$ & $30^{\circ} 16.6^{\prime} \mathrm{S}$ & $35^{\circ} 37.1^{\prime} \mathrm{W}$ & 1313 & CPDW, AAIW \\
\hline
\end{tabular}

Note: $\mathrm{AABW}=$ Antarctic Bottom Water; NADW $=$ North Atlantic Deep Water; CPDW $=$ Circumpolar Deep Water; AAIW $=$ Antarctic Intermediate Water.

\section{HYDROGRAPHY}

The physical oceanography of the western South Atlantic has been studied by Reid and others (1977). The western South Atlantic contains five distinct subsurface water masses that have originated from the cooling and sinking of surface waters at high latitudes (Fig. 2). The deepest water mass is Weddell Sea Deep Water (WSDW), which is formed in the Weddell Sea or farther east along the Antarctic continent. We have retained the more familiar term "AABW" in this paper to refer to WSDW. AABW is cold $\left(\theta<-0.4^{\circ} \mathrm{C}\right)$, highly saline $(\sim 34.7 \%)$, nutrient depleted, and oxygen enriched ( $>228 \mu \mathrm{M} / \mathrm{kg}$ ).
AABW is either overlain by a thin lens of lower Circumpolar Deep Water or directly overlain by North Atlantic Deep Water (NADW). Where NADW meets CPDW in the western South Atlantic, NADW splits CPDW into a lower and an upper branch; the narrower density range of NADW fits into the broader range of CPDW. CPDW is characterized by an oxygen minimum (as low as $\sim 200$ $\mu M / \mathrm{kg}$ ) and nutrient maximum. The low oxygen values of CPDW result from lateral exchange with deeper waters of the Indian and Pacific oceans while the relatively high salinity is derived from the North Atlantic (Reid et al., 1977). NADW originates in the Norwegian-Greenland Sea and is recognized by high salinities $(\sim 34.9 \%)$, 


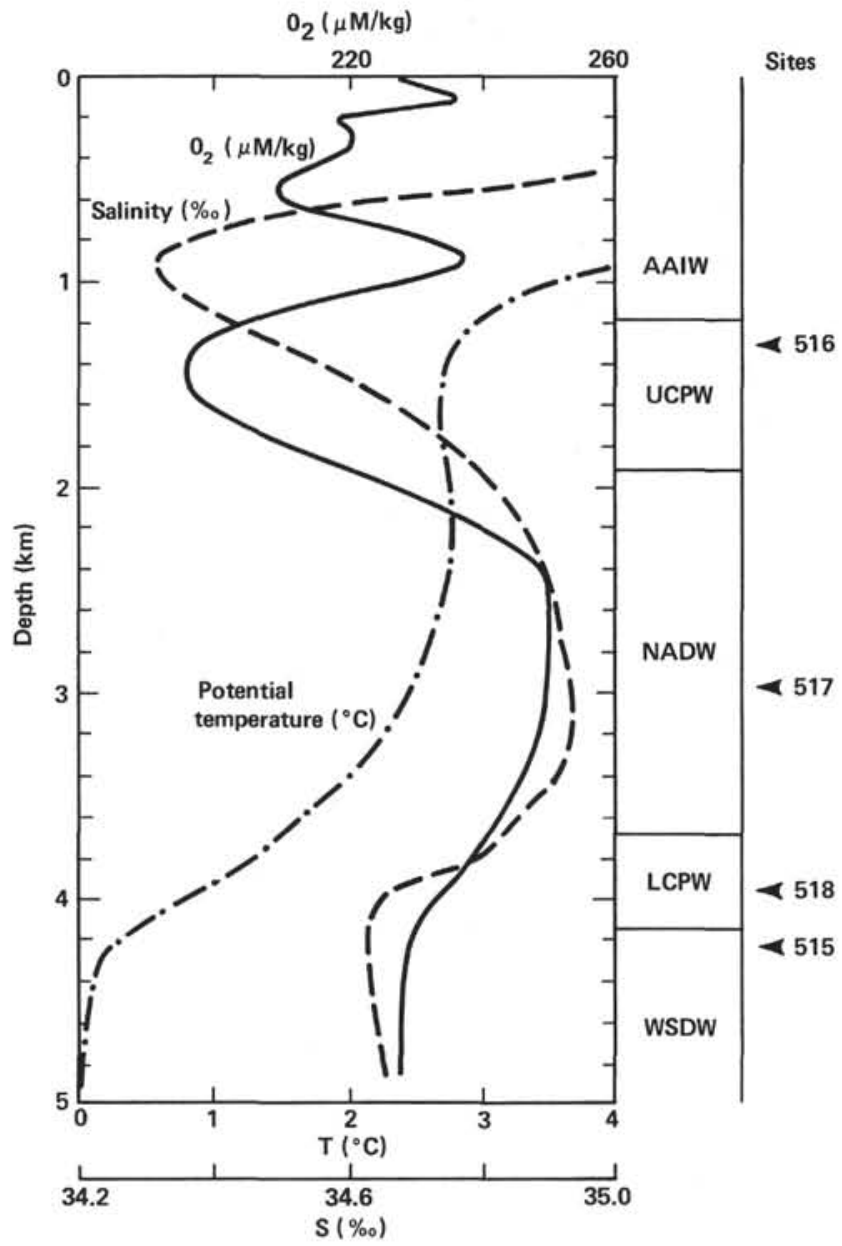

Figure 2. Position of Leg 72 sites relative to hydrographic data obtained from GEOSECS Station \#59 located in the axis of the Vema Channel. $A A I W=$ Antarctic Intermediate Water; $\mathrm{UCPW}=$ Upper Circumpolar Water; NADW $=$ North Atlantic Deep Water; LCPW $=$ Lower Circumpolar Water; WSDW $=$ Weddell Sea Deep Water or Antarctic Bottom Water. (After Barker et al., 1981.)

an oxygen maximum $(250 \mu \mathrm{M} / \mathrm{kg})$, and a nutrient minimum. NADW is overlain by the upper branch of CPDW. Antarctic Intermediate Water (AAIW) is the uppermost subsurface water mass and is formed by surface cooling at subantarctic latitudes.

\section{METHODS}

Quantitative counts of benthic foraminifers in the $>150 \mu \mathrm{m}$ size fraction were made on 34 samples from Site 518 (3944 m). Samples were oven dried at $50^{\circ} \mathrm{C}$, weighed, disaggregated in hot Calgon solution, washed over a $63 \mu \mathrm{m}$ Tyler screen, dried, and reweighed. Thirtyfive taxa were counted according to the taxonomy outlined by Corliss, 1979a (Table 2). No microsplitting of samples was necessary. The number of specimens counted per sample ranged from 107 to 1045 with an average of 319 counts per sample. Species percentages were calculated within the total population. Q-mode factor analysis was performed on the data set using the program of Klovan and Imbrie (1971).

Isotopic analyses were performed on Planulina wuellerstorfi and/ or Cibicidoides kullenbergi from Sites 517 and 518 (Tables 3, 4). Whenever possible, monospecific samples of $P$. wuellerstorfi were analyzed from the $>150 \mu \mathrm{m}$ size fraction, but the paucity of this species in some samples occasionally required using a mixture of $P$. wuellerstorfi and $C$. kullenbergi; however, these two species have been shown to be isotopically similar (Duplessy et al., 1980; Woodruff et al., 1981; Keigwin, 1982; and Leonard et al., this volume). Planktonic isotopic analyses were performed on Orbulina universa from Site 518 (Table 5) and on Globigerinoides quadrilobatus from Holes 516A and 517 (Leonard et al., this volume). Specimens for isotopic analysis were ultrasonically cleaned in deionized water, loaded into stainless steel carrying boats, and roasted in vacuo at $400^{\circ} \mathrm{C}$ for one hour to vaporize organic matter. The samples were then loaded into a reaction vessel and reacted to completion in $100 \%$ orthophosphoric acid at $50^{\circ} \mathrm{C}$. The resulting gas was distilled four times to remove water, and analyzed using an on-line VG Micromass 602D mass spectrometer. All isotopic results are expressed as per mil difference from PDB. Based upon replicate analyses of a powdered standard (B-1), analytic precision over a ten-month period was $\pm 0.10 \%$ for $\delta^{18} \mathrm{O}$ and $\pm 0.09 \%$ for $\delta^{13} \mathrm{C}$.

\section{STRATIGRAPHY}

Precise intersite correlation is essential in this study because benthic carbon isotopic records are directly compared between sites. The best stratigraphic control for Leg 72 is contained within the shallowest Hole 516A $(1313 \mathrm{~m})$, which has detailed magnetostratigraphy and biostratigraphy. Drilling at Hole 516A recovered a complete Pliocene section containing planktonic foraminiferal Zones PL1a through PL6 (Berggren et al., this volume; Pujol, this volume). The oldest sediment recovered at Site 517 is late Pliocene, and only Zones PL3 through PL6 are present (Berggren et al., this volume; Pujol, this volume). The Pliocene section of Site 518 extends from Zone PL1c through PL6 (Berggren et al., this volume; Pujol, this volume). A hiatus of approximately 1.5 Ma duration (5.5 to $4.0 \mathrm{Ma}$ ) spans the Miocene/ Pliocene boundary at Site 518, and Zones PL1a and PL1b are absent.

The late Pliocene sections of Site 517 and Hole 516A have been correlated using biostratigraphic datums and planktonic stable isotopic records (Leonard et al., this volume). The Pliocene of Site 518 is directly correlated with the chronology of Hole 516A in a similar manner (Fig. 3). The planktonic stable isotopic records of Site 518 and Hole 516A are in good agreement. Differences between the two records can be attributed to differing sedimentation rates. For example, shoaling of the lysocline is likely to decrease sedimentation rates at the deeper site (518) while producing no change at the shallower site $(516 \mathrm{~A})$. The correlation of the planktonic isotopic records also gives biostratigraphic datums that are generally consistent between Site 518 and Hole 516A. The isotopic correlations are more precise than those derived using the biostratigraphy because the sampling interval for isotopic analyses $(1 \mathrm{sample} / 50 \mathrm{~cm})$ is denser than the interval used to determine placement of biostratigraphic events (1 sample $/ 150 \mathrm{~cm}$ ).

\section{BENTHIC FORAMINIFERS}

One of the most recent applications of deep-sea benthic foraminiferal ecology has been to study the relationships between benthic foraminiferal distributions and hydrographic properties of subsurface water masses. Numerous quantitative core-top studies of benthic foraminiferal distributions have now been conducted in all oceans (Streeter, 1973; Schnitker, 1974; Culp, 1977; Gofas, 1978; Lohmann, 1978; Ingle et al., 1980; Resig, 1981). These studies form a basis for the extrapolation 
Table 2. Raw census data of 35 species of benthic foraminifers in the $>150 \mu \mathrm{m}$ fraction from DSDP Site 518 .

\begin{tabular}{|c|c|c|c|c|c|c|c|c|c|c|c|c|c|c|c|c|c|c|c|c|c|c|c|c|c|c|c|c|c|c|c|c|c|c|c|}
\hline $\begin{array}{l}\text { Sub-bottom } \\
\text { depth } \\
\text { (m) }\end{array}$ & 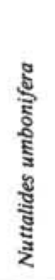 & 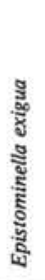 & 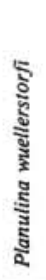 & $\begin{array}{l}0 \\
0 \\
0 \\
0 \\
0 \\
2 \\
2\end{array}$ & 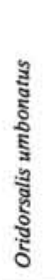 & 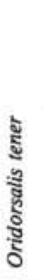 & 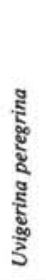 & 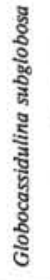 & 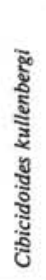 & 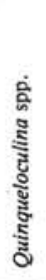 & 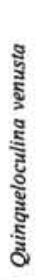 & 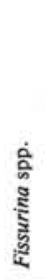 & 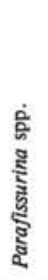 & 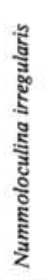 & 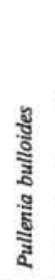 & 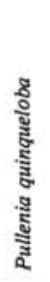 & $\begin{array}{l}\text { हิ } \\
\text { है } \\
\text { हैँ } \\
\text { हैँ } \\
\text { हैँ }\end{array}$ & 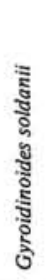 & 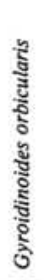 & 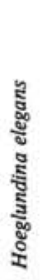 & $\begin{array}{l}\text { की } \\
\text { हूँ } \\
\text { ज्ञ } \\
\text { בे }\end{array}$ & $\begin{array}{l}\text { ฏ } \\
\text { है } \\
\text { है } \\
\text { ปี } \\
\text { हैँ } \\
\text { ปूँ }\end{array}$ & 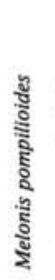 & 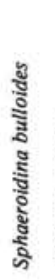 & $\begin{array}{l}\text { है } \\
\text { हूँ } \\
\text { हूँ } \\
\text { है }\end{array}$ & 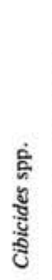 & 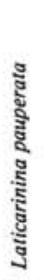 & 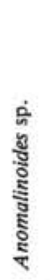 & 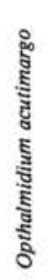 & 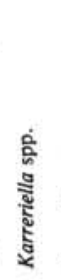 & 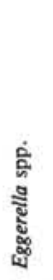 & 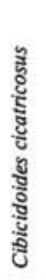 & 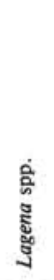 & 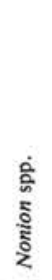 & \\
\hline 28.0 & 230 & 95 & 38 & 27 & 138 & 1 & 1 & 36 & 0 & 13 & 6 & 7 & 2 & 0 & 100 & 27 & 0 & 1 & 0 & 0 & 22 & 0 & 17 & 17 & 0 & & 0 & 0 & 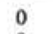 & 8 & 0 & 0 & 11 & 1 & \\
\hline & & 10 & 2 & 17 & 3 & 0 & 0 & & 2 & 3 & 5 & 2 & 0 & 0 & 24 & 14 & & 6 & & 0 & & & 10 & & & & & & & & & & 7 & 1 & \\
\hline 29.0 & 128 & 49 & 22 & 26 & 60 & 2 & 0 & 27 & 5 & 7 & 2 & 4 & 1 & 0 & 44 & 10 & 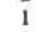 & 4 & 0 & 0 & 15 & 0 & 10 & 16 & & & & 0 & & 4 & 1 & & 5 & 1 & 2 \\
\hline 29.5 & 184 & 41 & 19 & 19 & 52 & 0 & 0 & 50 & 4 & 7 & 0 & 4 & 2 & 0 & 24 & 8 & c & 2 & 2 & 0 & 3 & ( & 5 & 13 & 0 & & & ( & & 5 & 1 & & 1 & 0 & 1 \\
\hline 30.0 & 524 & 96 & 18 & 10 & 160 & 0 & 0 & 10 & 6 & 3 & 0 & 14 & 0 & 0 & 90 & 21 & c & 3 & 1 & 0 & 14 & ( & 27 & & & & & & & 3 & 0 & & 4 & 0 & 2 \\
\hline 30.5 & 351 & 75 & 21 & 26 & 129 & 5 & 0 & 9 & 1 & 5 & 0 & 11 & 1 & 0 & 58 & 32 & 0 & 5 & 4 & 0 & 2 & 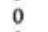 & 23 & & 0 & & & 0 & & 5 & 1 & 3 & 6 & 0 & 1 \\
\hline 31.0 & 67 & 38 & 16 & 19 & 32 & 9 & 0 & 8 & 1 & 0 & 0 & 3 & 1 & 0 & 29 & 6 & 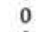 & 0 & 0 & 0 & 6 & 0 & 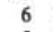 & 13 & 0 & & & 1 & & 2 & 1 & 4 & 10 & 0 & 0 \\
\hline 31. & 240 & 47 & 21 & 14 & 37 & 0 & 0 & 5 & 1 & 2 & 0 & 11 & 0 & 0 & 37 & 16 & c & 4 & 0 & 0 & 13 & 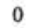 & 5 & & & & & ( & & & 0 & & 4 & 2 & 0 \\
\hline 31.9 & 52 & 29 & 23 & 19 & 54 & 0 & 0 & 51 & 3 & 0 & 0 & 3 & 1 & 0 & 16 & 14 & 0 & 3 & 2 & 0 & 3 & 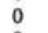 & 12 & 1 & 0 & & & 0 & & 4 & 0 & 5 & 1 & 1 & 1 \\
\hline 32.4 & 69 & 5 & 16 & 15 & 35 & 13 & 0 & 6 & 1 & 3 & 1 & 15 & 1 & 0 & 52 & 14 & ( & 8 & 2 & 0 & 8 & 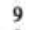 & 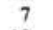 & & & & & & & 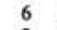 & 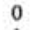 & & 1 & 0 & 2 \\
\hline 32.9 & 34 & 14 & 8 & 2 & 25 & 0 & 0 & 6 & 3 & 1 & 0 & 5 & 0 & 0 & 10 & 6 & 0 & 6 & 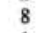 & 0 & & 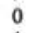 & 10 & & & & & ( & & & & & 0 & 0 & 0 \\
\hline 33.4 & 30 & 13 & 7 & 8 & 22 & 0 & 1 & 19 & 1 & 1 & 0 & 1 & 0 & 0 & 23 & 0 & 0 & 12 & & 0 & 0 & & & 5 & I & & & 1 & & 2 & 0 & 2 & 2 & 2 & 0 \\
\hline 33.9 & 70 & 1 & 10 & 4 & 25 & 0 & 7 & 26 & 9 & 2 & 4 & 2 & 0 & 0 & 24 & 12 & 1 & 6 & 2 & 0 & 3 & 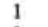 & 10 & 0 & 0 & & 0 & 1 & & & 2 & & 9 & 0 & 1 \\
\hline 34.4 & 79 & 4 & 7 & 19 & 25 & 0 & 39 & 42 & 14 & 4 & 0 & 11 & 0 & c & 27 & 16 & 0 & 7 & 11 & 0 & 4 & & & & 12 & & & 0 & & 7 & 0 & 3 & 5 & 7 & 0 \\
\hline 36.3 & 55 & 1 & 9 & 11 & 25 & 6 & 2 & 26 & 9 & 2 & 1 & 8 & 0 & & 34 & 1 & 2 & 12 & & 0 & 4 & & 3 & 5 & & & 3 & 3 & & 4 & 0 & 4 & 8 & 15 & 2 \\
\hline 36.8 & 38 & 0 & 11 & 1 & 13 & 1 & 14 & 2 & 9 & 1 & 12 & 0 & 0 & & 21 & 16 & ( & 6 & 7 & & 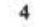 & & 0 & & & & & 1 & & & c & & 4 & & 0 \\
\hline & 2 & 1 & 10 & 8 & 18 & 2 & 10 & 23 & 7 & 1 & 3 & 3 & 0 & c & 7 & 7 & & 2 & 3 & & ( & & 4 & & & & & 0 & & 7 & 2 & 1 & 3 & 3 & 0 \\
\hline 37.8 & 45 & 0 & 6 & 4 & 12 & 1 & 6 & 35 & 5 & 4 & 0 & 7 & 1 & 0 & 9 & 11 & c & 2 & 2 & 0 & & & 3 & 0 & 1 & 1 & & 1 & & 3 & 0 & & 1 & $c$ & 0 \\
\hline 38.2 & 80 & 0 & 6 & 5 & 8 & 3 & 7 & 3 & 9 & 2 & c & 6 & c & C & 12 & 12 & 0 & 4 & ( & & 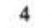 & & 2 & & & & & 3 & & 9 & 0 & & 6 & 8 & 0 \\
\hline & 1 & 0 & 5 & 6 & 43 & 0 & 0 & 25 & 4 & 2 & 1 & 5 & 2 & 0 & 10 & 4 & 4 & 9 & 0 & & 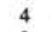 & ( & & & & & & 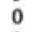 & & 0 & 1 & & 2 & 0 & 0 \\
\hline & 49 & 8 & 6 & 4 & 29 & 1 & 0 & 2 & 6 & 1 & 0 & & 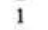 & & 13 & & 4 & 10 & 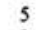 & & & 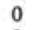 & 1 & & 0 & 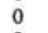 & & ( & ( & 7 & 0 & & 4 & 3 & 2 \\
\hline 41.7 & 51 & 15 & 13 & 4 & 65 & 0 & 0 & 5 & 3 & 1 & 0 & 1 & c & & 10 & 13 & 0 & 8 & 3 & 0 & & 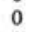 & 25 & 13 & 0 & & & 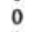 & ( & 11 & 0 & & 2 & 3 & 3 \\
\hline & 3 & 0 & 10 & 6 & 34 & 0 & 0 & 27 & 6 & 1 & 1 & i & 0 & & 8 & 6 & 0 & 10 & & & & 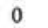 & & & & & & 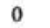 & & & & 3 & 3 & & 2 \\
\hline & $3 c$ & 2 & 12 & 3 & 1 & 3 & 0 & 22 & 5 & 3 & 0 & S & c & & 13 & & 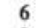 & 5 & & & & ( & 13 & & & ( & & ( & ( & 3 & 0 & 4 & 3 & 0 & 0 \\
\hline 43.2 & 25 & 0 & 1 & 2 & 20 & 0 & 0 & 7 & 5 & 2 & 3 & 3 & c & & 5 & 4 & 2 & 4 & 2 & & & c & 1 & & & & & 0 & c & 1 & 0 & & 2 & 0 & 1 \\
\hline & 118 & 0 & 2 & 8 & 8 & 0 & 0 & 9 & 5 & 0 & 0 & & c & & 6 & 10 & 2 & 3 & & c & 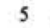 & & 3 & & & & & ( & c & 1 & 1 & & 5 & 0 & 0 \\
\hline & 24 & 11 & 12 & 4 & 45 & 0 & 1 & 7 & 2 & 3 & 0 & & 0 & & 8 & & & $\epsilon$ & & & & & 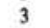 & 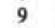 & & & & & ( & 2 & 0 & 0 & 3 & 1 & 0 \\
\hline 4 & 5 & 2 & 16 & 6 & 40 & 1 & 31 & 54 & 5 & 0 & 1 & 8 & 1 & 1 & 12 & 5 & 7 & 7 & 9 & 0 & 11 & 3 & 6 & 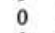 & 3 & 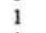 & 2 & 1 & 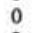 & 0 & 0 & 4 & 1 & 1 & 0 \\
\hline & 3 & 1 & 14 & 2 & 1 & 0 & 2 & 4 & 1 & 0 & 0 & 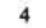 & 0 & 0 & 8 & & 4 & 10 & 6 & 1 & 5 & 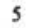 & 0 & c & r & 1 & & c & & 1 & & 8 & 3 & & \\
\hline & 2 & 1 & 10 & 1 & 25 & 2 & 33 & 5 & 17 & 1 & 1 & 3 & 0 & & 9 & & 13 & 13 & & & & 8 & & & & & & & c & 5 & 0 & 4 & 4 & 3 & 0 \\
\hline & 27 & 1 & 6 & 4 & 19 & 1 & 16 & 2 & 5 & 0 & 0 & 6 & 1 & 0 & 10 & 5 & 5 & 3 & 2 & c & & 3 & 2 & 1 & 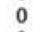 & c & 2 & 2 & c & 1 & 0 & 3 & 4 & 3 & 0 \\
\hline & 9 & 0 & 1 & 3 & 1 & 0 & 0 & 2 & 1 & 1 & 0 & 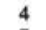 & 5 & & 1 & 3 & 4 & 13 & 7 & & & c & 3 & c & ( & & & & & 0 & & 5 & 0 & 1 & \\
\hline 51.0 & 29 & 1 & 10 & 6 & 22 & 2 & 25 & 23 & 14 & 0 & 0 & 7 & 2 & 0 & & 6 & 2 & 15 & & & & 0 & 5 & 0 & 1 & 0 & 0 & 0 & 0 & 1 & 0 & 1 & 3 & 1 & 0 \\
\hline 53.9 & 39 & 1 & 4 & 3 & 19 & 0 & 17 & 22 & 10 & 0 & 0 & 2 & 0 & 0 & 11 & 8 & 4 & 7 & 10 & 0 & 0 & 0 & 4 & 4 & 2 & 2 & 1 & 0 & 1 & 0 & 0 & 7 & 4 & 2 & 0 \\
\hline
\end{tabular}

Table 3. Oxygen and carbon isotopic results (in \% PDB) of Planulina wuellerstorfi and/or Cibicidoides kullenbergi from DSDP Site 517.

\begin{tabular}{lcccc}
\hline $\begin{array}{c}\text { Core-section } \\
\text { (interval in cm) }\end{array}$ & $\begin{array}{c}\text { Depth } \\
\text { (m) }\end{array}$ & $\begin{array}{c}\mathrm{Age}^{\mathrm{a}} \\
\text { (Ma) }\end{array}$ & $\delta^{18} \mathrm{O}$ & ${ }^{13} \mathrm{C}$ \\
\hline $9-3,20-22$ & 36.5 & 2.81 & 2.58 & 1.11 \\
$10-2,20-22$ & 37.9 & 2.96 & 2.36 & 1.06 \\
$10-2,120-122$ & 38.9 & 2.99 & 2.37 & 1.08 \\
$10-3,70-72$ & 41.4 & 3.01 & 2.39 & 0.87 \\
$11-2,20-22$ & 43.8 & 3.07 & 3.00 & 0.26 \\
$11-2,70-72$ & 44.3 & 3.09 & 2.31 & 1.06 \\
$11-2,120-122$ & 44.8 & 3.10 & 2.22 & 1.03 \\
$11-3,20-22$ & 45.3 & 3.11 & 2.22 & 0.97 \\
$11-3,70-72$ & 45.8 & 3.13 & 2.13 & 0.97 \\
$12-1,120-122$ & 47.7 & 3.18 & 2.42 & 0.91 \\
$12-2,20-22$ & 48.2 & 3.19 & 2.67 & 0.82 \\
$12-2,70-72$ & 48.7 & 3.20 & 2.43 & 0.86 \\
$12-2,120-122$ & 49.2 & 3.21 & 2.24 & 0.87 \\
$12-3,20-22$ & 49.7 & 3.23 & 2.17 & 1.17 \\
$12-3,120-122$ & 50.7 & 3.25 & 2.04 & 0.62 \\
\hline
\end{tabular}

a Age assignments have been rounded to the nearest 10,000 years.

of modern assemblage-water mass relationships into the geologic past. Although modern studies have shown that benthic foraminiferal distributions tend to be controlled by specific water masses, it has been difficult to determine which of the many hydrographic properties (temperature, alkalinity, silica, phosphate, nitrate, $\Sigma \mathrm{CO}_{2}$, etc.) is actually controlling their distribution.
This problem results from the fact that hydrographic properties tend to be highly intercorrelated. Nevertheless, quantitative analyses of benthic foraminifers still provide one of the best methods for studying the distribution of subsurface water masses in the past.

Factor analysis of 34 samples from Site 518 reveals three distinct benthic foraminiferal assemblages, which account for $92 \%$ of the total variance (Tables 6 and 7). Factor 1 is heavily dominated by Nuttalides umbonifera; Factor 2 consists of a Globocassidulina subglobosa-Uvigerina peregrina assemblage; and Factor 3 is largely composed of an Oridorsalis umbonatus-Epistominella exigua assemblage.

Changes in these benthic foraminiferal assemblages during the Pliocene of Site 518 are marked by three distinct events (Fig. 4). From 4.1 to $3.6 \mathrm{Ma}$, the N. umbonifera (Factor 1) and O. umbonatus-E. exigua (Factor 3) assemblages dominate. At 3.6 Ma, these assemblages are replaced by the $G$. subglobosa-U. peregrina (Factor 2) assemblage. This change at $3.6 \mathrm{Ma}$ is best illustrated by the abundance of $N$. umbonifera, $U$. peregrina, and E. exigua (Fig. 5). Between 3.6 and $3.2 \mathrm{Ma}$, $U$. peregrina increases to $10 \%, E$. exigua decreases to $<1 \%$, and $N$. umbonifera averages $20 \%$. The most dramatic shift in benthic foraminiferal assemblages occurs at $3.2 \mathrm{Ma}$ when the $G$. subglobosa-U. peregrina assemblage (Factor 2) is replaced by the $N$. umbonifera (Factor 1 ) and $O$. umbonatus-E. exigua (Factor 3 ) assemblages. $U$. peregrina disappears $(0 \%), E$. exigua perma- 
Table 4. Oxygen and carbon isotopic results (in \% PDB) of Planulina wuellerstorfi and/or Cibicidoides kullenbergi from Site 518.

\begin{tabular}{|c|c|c|c|c|}
\hline $\begin{array}{l}\text { Core-section } \\
\text { (interval in cm) }\end{array}$ & $\begin{array}{l}\text { Depth } \\
\text { (m) }\end{array}$ & $\begin{array}{l}\mathrm{Age}^{\mathrm{a}} \\
(\mathrm{Ma})\end{array}$ & $\delta^{18} \mathrm{O}$ & $\delta^{13} \mathrm{C}$ \\
\hline $8-1,70-72$ & 28.0 & 2.79 & 3.08 & 0.50 \\
\hline $8-1,120-122$ & 28.5 & 2.83 & 2.73 & 0.43 \\
\hline $8-2,20-22$ & 29.0 & 2.87 & 2.95 & -0.02 \\
\hline $8-2,70-72$ & 29.5 & 2.91 & 2.63 & 0.56 \\
\hline $8-2,120-122$ & 30.0 & 2.95 & 3.21 & 0.40 \\
\hline $8-3,20-22$ & 30.5 & 2.99 & 2.98 & 0.33 \\
\hline $8-3,70-72$ & 31.0 & 3.03 & 2.71 & 0.27 \\
\hline $8-3,120-122$ & 31.5 & 3.08 & 2.75 & 0.44 \\
\hline $9-1,20-22$ & 31.9 & 3.11 & 2.48 & 0.66 \\
\hline $9-1,70-72$ & 32.4 & 3.16 & 2.75 & 0.65 \\
\hline $9-1,120-122$ & 32.9 & 3.20 & 2.08 & 0.86 \\
\hline $9-2,10-14$ & 33.3 & 3.24 & 2.19 & 0.75 \\
\hline $9-2,40-44$ & 33.6 & 3.26 & 2.14 & 0.82 \\
\hline $9-2,70-72$ & 33.9 & 3.29 & 2.41 & 0.53 \\
\hline $9-2,90-94$ & 34.1 & 3.33 & 2.09 & 1.10 \\
\hline $9-2,120-122$ & 34.4 & 3.33 & 2.39 & 0.96 \\
\hline $9-3,10-14$ & 34.8 & 3.37 & 2.08 & 1.03 \\
\hline $10-1,70-72$ & 36.8 & 3.55 & 2.32 & 1.00 \\
\hline $10-1,120-122$ & 37.3 & 3.59 & 2.38 & 0.96 \\
\hline $10-2,20-22$ & 37.8 & 3.63 & 2.16 & 0.92 \\
\hline $10-2,51-55$ & 38.1 & 3.66 & 2.13 & 1.04 \\
\hline $10-2,59-61$ & 38.2 & 3.66 & 2.16 & 1.00 \\
\hline $10-2,90-94$ & 38.5 & 3.67 & 2.12 & 1.17 \\
\hline $11-1,10-14$ & 40.6 & 3.72 & 2.13 & 0.74 \\
\hline $11-1,68-72$ & 41.2 & 3.79 & 2.44 & 0.80 \\
\hline $11-1,117-122$ & 41.7 & 3.84 & 2.50 & 0.59 \\
\hline $11-2,20-22$ & 42.2 & 3.89 & 2.50 & 0.89 \\
\hline $11-2,40-44$ & 42.4 & 3.92 & 2.37 & 0.97 \\
\hline $11-2,70-72$ & 42.7 & 3.95 & 2.28 & 0.73 \\
\hline $11-2,120-122$ & 43.2 & 4.00 & 2.39 & 0.74 \\
\hline $11-2,130-134$ & 43.3 & 4.01 & 2.23 & 0.30 \\
\hline $11-3,19-21$ & 43.7 & 4.06 & 2.56 & 0.83 \\
\hline $11-3,31-35$ & 43.8 & 4.07 & 2.12 & 0.71 \\
\hline $11-3,50-52$ & 44.0 & 4.09 & 2.36 & 0.76 \\
\hline
\end{tabular}

nently increases to $10 \%$, and $N$. umbonifera increases by $30 \%$.

In order to interpret these assemblage changes in terms of water masses, the Pliocene assemblages must be compared with Recent core top studies. Lohmann $(1978,1981)$ studied benthic foraminiferal distributions $(>250 \mu \mathrm{m})$ from the Rio Grande Rise, and recognized four taxonomically distinct assemblages which coincide with the distribution of subsurface water masses: (1) a Nuttalides umbonifera assemblage $(>3500 \mathrm{~m})$ coinciding with AABW; (2) a miliolid-cibicid-Oridorsalis assemblage ( 2100 to $3500 \mathrm{~m}$ ) coinciding with NADW; (3) a Uvigerina assemblage (1500 to $2200 \mathrm{~m}$ ) coinciding with CPDW; (4) a Hoeglundina-Globocassidulina assemblage ( 800 to $1500 \mathrm{~m}$ ) coinciding with AAIW. Unfortunately, these assemblage-water mass relationships cannot be directly applied to the Site 518 data, owing to a difference in size fraction. Lohmann $(1978,1981)$ collected data at the $>250 \mu \mathrm{m}$ size fraction, while counts for the Site 518 samples were made at the $>150 \mu \mathrm{m}$ size fraction. Although the results of Lohmann $(1978,1981)$ are not directly applicable, some similarities do exist.
Table 5. Oxygen and carbon isotopic results (in \%。 PDB) of Orbulina universa from DSDP Site 518.

\begin{tabular}{|c|c|c|c|c|}
\hline $\begin{array}{l}\text { Core-section } \\
\text { (interval in cm) }\end{array}$ & $\begin{array}{l}\text { Depth } \\
\text { (m) }\end{array}$ & $\begin{array}{l}\mathrm{Age}^{\mathrm{a}} \\
(\mathrm{Ma})\end{array}$ & $\delta^{18} \mathrm{O}$ & $\delta^{13} \mathrm{C}$ \\
\hline $8-1,70-72$ & 28.0 & 2.79 & 0.54 & 2.21 \\
\hline $8-1,120-122$ & 28.5 & 2.83 & 0.19 & 2.36 \\
\hline $8-2,20-22$ & 29.0 & 2.87 & 0.53 & 2.02 \\
\hline $8-2,70-72$ & 29.5 & 2.91 & 0.31 & 1.96 \\
\hline $8-3,20-22$ & 30.5 & 2.99 & 0.72 & 2.21 \\
\hline $8-3,70-72$ & 31.0 & 3.03 & 0.32 & 2.49 \\
\hline $8-3,120-122$ & 31.5 & 3.08 & 0.62 & 2.53 \\
\hline $9-1,20-22$ & 31.9 & 3.11 & 0.30 & 2.40 \\
\hline $9-1,70-72$ & 32.4 & 3.16 & 0.70 & 2.23 \\
\hline $9-1,120-122$ & 32.9 & 3.20 & 0.40 & 1.98 \\
\hline $9-2,10-14$ & 33.3 & 3.24 & 0.39 & 1.73 \\
\hline $9-2,20-22$ & 33.4 & 3.25 & -0.09 & 2.14 \\
\hline $9-2,40-44$ & 33.6 & 3.26 & 0.43 & 1.89 \\
\hline $9-2,70-72$ & 33.9 & 3.29 & 0.37 & 2.19 \\
\hline $9-2,90-94$ & 34.1 & 3.31 & -0.02 & 2.30 \\
\hline $9-2,120-122$ & 34.4 & 3.33 & 0.80 & 2.60 \\
\hline $9-3,10-14$ & 34.8 & 3.37 & 0.53 & 1.85 \\
\hline $10-1,20-22$ & 36.3 & 3.50 & 0.66 & 1.83 \\
\hline $10-1,40-44$ & 36.5 & 3.52 & 0.22 & 2.01 \\
\hline $10-1,70-72$ & 36.8 & 3.55 & -0.09 & 2.07 \\
\hline $10-1,120-122$ & 37.3 & 3.59 & 0.24 & 2.05 \\
\hline $10-2,20-22$ & 37.8 & 3.63 & 0.63 & 2.26 \\
\hline $10-2,51-55$ & 38.1 & 3.66 & 0.30 & 2.12 \\
\hline $10-2,59-61$ & 38.2 & 3.66 & 0.32 & 2.48 \\
\hline $11-1,10-14$ & 40.6 & 3.72 & 0.81 & 2.03 \\
\hline $11-1,20-23$ & 40.7 & 3.73 & -0.16 & 1.88 \\
\hline $11-1,68-72$ & 41.2 & 3.79 & 0.49 & 1.82 \\
\hline $11-1,117-122$ & 41.7 & 3.84 & 0.28 & 1.38 \\
\hline $11-2,20-22$ & 42.2 & 3.89 & 0.07 & 1.67 \\
\hline $11-2,40-44$ & 42.4 & 3.92 & 0.49 & 1.59 \\
\hline $11-2,70-72$ & 42.7 & 3.95 & 0.35 & 1.88 \\
\hline $11-2,90-94$ & 42.9 & 3.97 & 0.64 & 1.71 \\
\hline $11-2,120-122$ & 43.2 & 4.00 & 0.61 & 1.95 \\
\hline $11-2,130-134$ & 43.3 & 4.01 & 0.56 & 1.77 \\
\hline $11-3,19-21$ & 43.7 & 4.06 & 0.45 & 2.15 \\
\hline
\end{tabular}

The $N$. umbonifera assemblage of our study (Factor $1)$ is identical to that of Lohmann's $(1978,1981)$. The preference of this species for AABW has been demonstrated in all oceans (Streeter, 1973; Schnitker, 1974; Lohmann, 1978, 1981; Gofas, 1978; Corliss, 1978a, $1978 \mathrm{~b}, 1979 \mathrm{~b}, 1983$. The association of dominant $N$. umbonifera with AABW is partly the result of this species' tolerance for intense carbonate undersaturation as compared with other species (Bremer and Lohmann, 1982; Corliss, 1983).

Lohmann's (1981) miliolid-cibicid-Oridorsalis assemblage may coincide with Factor 3 (O. umbonatus-E. exigua) from this study, thereby indicating NADW. Lohmann (1981) didn't record E. exigua in his samples because the average largest diameter of this species is about 100 to $120 \mu \mathrm{m}$ (Schnitker, 1980). However, a cursory check by Schnitker (personal communication) showed $E$. exigua to be present in Rio Grande Rise core tops. Evidence that Factor $3(O$. umbonatus-E. exigua $)$ is associated with NADW comes from Schnitker (1974) who found an E. exigua assemblage associated with Norwegian Sea Outflow Water. The most important accessory 


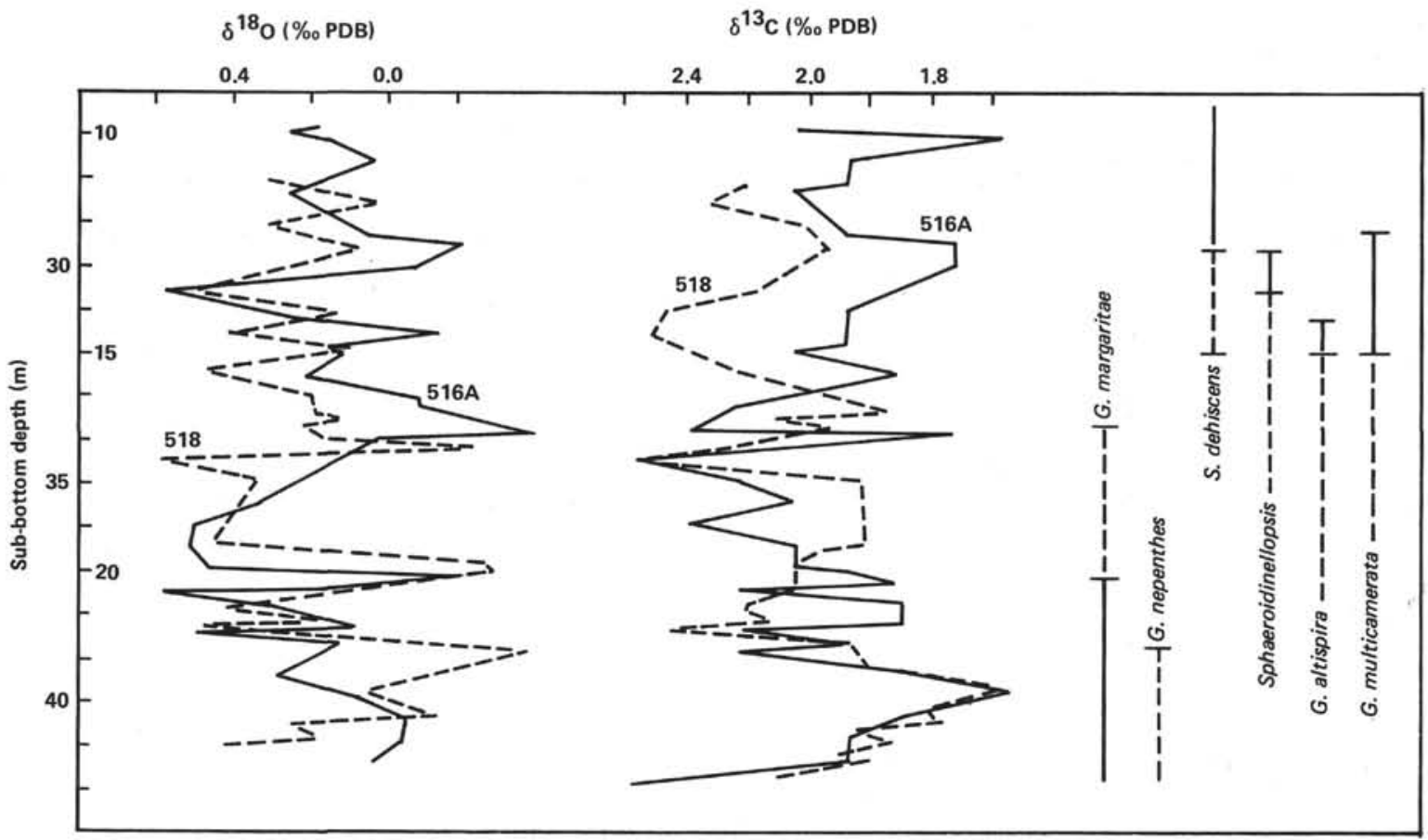

Figure 3. Isotopic and biostratigraphic correlation between DSDP Hole 516A and Site 518. Isotopic data include oxygen and carbon isotopes from Hole 516A (solid line) run on Globigerinoides sacculifera (Leonard et al., this volume) and from Site 518 run on Orbulina universa (this study). Biostratigraphic data include first and last appearances of key planktonic foraminifers from Hole 516A (solid line) and Site 518 (dashed line) (Berggren et al., this volume; Pujol, this volume). G. = Globorotalia; $S$. = Sphaeroidinella.

species in this assemblage was $O$. umbonatus. In the South Atlantic, this assemblage differs in that $E$. exigua decreases in importance while $O$. umbonatus becomes dominant. This increased dominance of $O$. umbonatus over E. exigua at $30^{\circ} \mathrm{S}$ may be the result of increased mixing as NADW travels south.

Lastly, the $G$. subglobosa-U. peregrina assemblage (Factor 2) of our study may correspond to either the Uvigerina (CPDW) or Globocassidulina (AAIW) assemblage of Lohmann (1981). Corliss (1983) reports a G. subglobosa assemblage from the southeast Indian Ocean which is associated with a mixture of AABW and CPDW with potential temperatures of 0.6 to $0.8^{\circ} \mathrm{C}$. Schnitker (1974) also described a G. subglobosa-U. peregrina assemblage along the continental margin and mid-ocean ridge of the North Atlantic. This assemblage is associated with NADW with potential temperatures between 2 and $4^{\circ} \mathrm{C}$. We interpret the $G$. subglobosa- $U$. peregrina (Factor 2) assemblage in Site 518 as indicating a shallower water mass of circumpolar origin. This water mass may have had slightly different physical and chemical properties than modern CPDW. For example, Corliss (1982) has presented benthic foraminiferal evidence for "glacial" CPDW which has distinctly different properties than its modern counterpart.

In summary, benthic foraminifers from the late Neogene of Site 518 can be grouped into three assemblages which are indicative of specific water masses in the geologic past (Fig. 4). These assemblages include: an $N$. umbonifera assemblage associated with AABW, a $G$. subglobosa-U. peregrina assemblage associated with
Table 6. Scaled varimax factor scores for each of the 35 taxa recorded in this study. The factor scores indicate the relative contribution of each of the benthic foraminiferal taxa to the five factor assemblages.

\begin{tabular}{|c|c|c|c|c|c|}
\hline \multirow[b]{2}{*}{ Species } & \multicolumn{5}{|c|}{ Factor assemblage } \\
\hline & 1 & 2 & 3 & 4 & 5 \\
\hline Nuttalides umbonifera & 5.661 & 1.195 & -0.098 & -0.731 & -0.164 \\
\hline Epistominella exigua & 0.783 & -1.009 & 1.721 & 1.302 & -1.202 \\
\hline Planulina wuellerstorfi & 0.121 & 0.533 & 0.593 & 0.154 & 2.371 \\
\hline Pyrgo spp. & 0.280 & 0.158 & 0.473 & 1.022 & 0.159 \\
\hline Oridorsalis umbonatus & -0.288 & 0.609 & 5.171 & 0.370 & -1.234 \\
\hline Oridorsalis tener & 0.061 & 0.065 & -0.098 & 0.712 & 0.302 \\
\hline Uvigerina peregrina & -0.575 & 3.104 & -1.394 & 2.445 & -3.286 \\
\hline Globocassidulina subglobosa & -0.870 & 4.140 & 0.741 & -2.460 & 0.687 \\
\hline Cibicidoides kullenbergi & -0.380 & 1.380 & -0.366 & 0.224 & 1.437 \\
\hline Quinquelocina spp. & 0.087 & -0.011 & 0.160 & -0.046 & 0.214 \\
\hline Quinqueloculina venusta & 0.038 & 0.171 & -0.120 & 0.217 & 0.194 \\
\hline Fissurina spp. & 0.024 & 0.385 & 0.292 & -0.158 & 0.769 \\
\hline Parafissurina spp. & -0.103 & 0.034 & 0.058 & 0.035 & 0.728 \\
\hline Nummoloculina irregularis & -0.002 & 0.009 & 0.001 & -0.005 & -0.022 \\
\hline Pullenia bulloides & 0.627 & 0.532 & 0.284 & 4.003 & 2.435 \\
\hline Pullenia quinqueloba & 0.325 & 0.789 & -0.048 & 0.407 & 0.185 \\
\hline Ehrenbergina spp. & -0.237 & 0.501 & 0.090 & -0.177 & 0.183 \\
\hline Gyroidinoides sodanii & -0.480 & 0.744 & 0.459 & 0.797 & 1.924 \\
\hline Gyroidinoides orbicularis & -0.170 & 0.676 & -0.179 & 0.713 & 0.606 \\
\hline Hoeglundina elegans & -0.019 & 0.047 & -0.001 & -0.018 & -0.023 \\
\hline Pullenia sp. & 0.126 & 0.249 & 0.100 & 0.283 & -0.113 \\
\hline Osangularia culter & -0.048 & 0.261 & -0.155 & 0.412 & -0.016 \\
\hline Melonis pompiloides & 0.108 & -0.027 & 0.904 & -0.634 & 0.876 \\
\hline Sphaeroidina bulloides & 0.165 & -0.262 & 0.776 & 0.320 & -0.421 \\
\hline Bulimina rostrata & 0.011 & 0.309 & -0.250 & 0.525 & -0.290 \\
\hline Cibicides spp. & -0.015 & 0.124 & -0.007 & -0.057 & -0.154 \\
\hline Laticarinina pauperata & -0.034 & 0.108 & 0.006 & 0.030 & 0.019 \\
\hline Anomalinoides sp. & 0.015 & 0.090 & -0.089 & 0.087 & 0.139 \\
\hline Opthalmidium acutimargo & -0.008 & 0.046 & -0.022 & -0.010 & -0.029 \\
\hline Karreriella spp. & 0.072 & 0.261 & 0.122 & -0.171 & 0.103 \\
\hline Eggerella spp. & 0.004 & 0.007 & 0.057 & -0.041 & 0.009 \\
\hline Cibicidoides cicatricosus & -0.105 & 0.359 & 0.100 & -0.169 & 0.606 \\
\hline Lagena spp. & 0.138 & 0.334 & -0.032 & 0.382 & -0.162 \\
\hline Nonion spp. & -0.025 & 0.455 & -0.241 & 0.533 & -0.147 \\
\hline Lenticulina spp. & -0.007 & -0.028 & 0.084 & -0.039 & 0.383 \\
\hline
\end{tabular}


Table 7. Varimax factor loadings of the first five factors of 34 samples from the late Neogene of Site 518. Only the first three factors, which explain $\sim 92 \%$ of the total variance, were used in this study.

\begin{tabular}{|c|c|c|c|c|c|c|}
\hline \multirow{2}{*}{$\begin{array}{l}\text { Sub-bottom } \\
\text { depth } \\
\text { (m) }\end{array}$} & \multirow[b]{2}{*}{ Comm. } & \multicolumn{5}{|c|}{ Factor } \\
\hline & & 1 & 2 & 3 & 4 & 5 \\
\hline 28.0 & 0.984 & 0.760 & 0.285 & 0.531 & 0.201 & 0.033 \\
\hline 28.5 & 0.978 & 0.843 & 0.382 & 0.316 & 0.036 & 0.141 \\
\hline 29.0 & 0.977 & 0.787 & 0.323 & 0.478 & 0.150 & 0.054 \\
\hline 29.5 & 0.984 & 0.855 & 0.377 & 0.330 & -0.049 & 0.014 \\
\hline 30.0 & 0.986 & 0.914 & 0.226 & 0.311 & 0.047 & -0.026 \\
\hline 30.5 & 0.992 & 0.893 & 0.235 & 0.367 & 0.062 & -0.026 \\
\hline 31.0 & 0.937 & 0.759 & 0.236 & 0.487 & 0.257 & 0.053 \\
\hline 31.5 & 0.992 & 0.947 & 0.226 & 0.203 & 0.046 & 0.014 \\
\hline 31.9 & 0.936 & 0.457 & 0.525 & 0.668 & -0.021 & 0.077 \\
\hline 32.4 & 0.940 & 0.707 & 0.328 & 0.388 & 0.338 & 0.261 \\
\hline 32.9 & 0.948 & 0.671 & 0.329 & 0.602 & 0.131 & 0.096 \\
\hline 33.4 & 0.928 & 0.544 & 0.478 & 0.555 & 0.231 & 0.206 \\
\hline 33.9 & 0.976 & 0.746 & 0.549 & 0.307 & 0.050 & 0.143 \\
\hline 34.4 & 0.980 & 0.615 & 0.733 & 0.194 & 0.167 & -0.018 \\
\hline 36.3 & 0.970 & 0.555 & 0.699 & 0.246 & 0.327 & 0.076 \\
\hline 36.8 & 0.932 & 0.536 & 0.743 & 0.197 & 0.166 & 0.166 \\
\hline 37.3 & 0.944 & 0.379 & 0.780 & 0.429 & 0.030 & 0.088 \\
\hline 37.8 & 0.970 & 0.620 & 0.704 & 0.238 & -0.141 & 0.118 \\
\hline 38.2 & 0.983 & 0.789 & 0.574 & 0.110 & -0.093 & 0.094 \\
\hline 40.7 & 0.950 & 0.200 & 0.546 & 0.776 & -0.011 & 0.100 \\
\hline 41.2 & 0.961 & 0.654 & 0.501 & 0.507 & -0.007 & 0.160 \\
\hline 41.7 & 0.969 & 0.375 & 0.570 & 0.693 & -0.140 & 0.064 \\
\hline 42.2 & 0.972 & 0.493 & 0.595 & 0.584 & -0.087 & 0.163 \\
\hline 42.7 & 0.949 & 0.597 & 0.567 & 0.448 & -0.034 & 0.264 \\
\hline 43.2 & 0.887 & 0.627 & 0.455 & 0.532 & 0.012 & 0.064 \\
\hline 43.7 & 0.969 & 0.922 & 0.309 & 0.083 & -0.085 & 0.100 \\
\hline 44.0 & 0.956 & 0.399 & 0.305 & 0.827 & 0.139 & -0.019 \\
\hline 45.3 & 0.973 & 0.437 & 0.794 & 0.387 & -0.015 & -0.029 \\
\hline 45.8 & 0.970 & 0.266 & 0.909 & 0.249 & -0.044 & 0.093 \\
\hline 49.5 & 0.972 & 0.095 & 0.935 & 0.299 & 0.014 & 0.003 \\
\hline 50.0 & 0.981 & 0.409 & 0.824 & 0.363 & 0.045 & 0.002 \\
\hline 50.5 & 0.959 & 0.108 & 0.687 & 0.443 & 0.072 & 0.524 \\
\hline 51.0 & 0.954 & 0.360 & 0.808 & 0.328 & 0.239 & 0.081 \\
\hline 53.9 & 0.959 & 0.561 & 0.739 & 0.297 & 0.099 & 0.020 \\
\hline Variance & & 39.363 & 33.359 & 19.586 & 1.919 & 2.000 \\
\hline Cumulative $\mathrm{V}$ & ariance & 39.363 & 72.722 & 92.308 & 94.227 & 96.227 \\
\hline
\end{tabular}

CPDW, and an O. umbonatus-E. exigua assemblage associated with NADW.

\section{BENTHIC OXYGEN ISOTOPIC RECORDS}

Variations in the oxygen isotopic composition of foraminiferal tests are known to reflect changes in salinity, temperature, and ice volume. Although the controls on the $\delta^{18} \mathrm{O}$ record are well understood, the degree to which each affects the record is often debated.

The benthic $\delta^{18} \mathrm{O}$ record of Site 518 is relatively stable until the late Pliocene. At 3.2 Ma, a large enrichment of $\sim 1.1 \%$ peak ( $0.45 \%$ average) occurs (Fig. 6$)$. The benthic $\delta^{18} \mathrm{O}$ record of Site 517 undergoes a $0.8 \%$ steplike enrichment between 3.3 and 2.8 Ma (Fig. 7). In Hole $516 \mathrm{~A}$, a $0.5 \%$ enrichment is also observed at this time (Leonard et al., this volume). Therefore, a benthic ${ }^{18} \mathrm{O}$ enrichment is observed in all Leg 72 sites at 3.2 Ma, and the magnitude of the enrichment appears to increase from Hole 516A (1313 m water depth, $0.5 \%$ ) to Site 517 (2963 m water depth, $0.8 \%$ ) to Site 518 (3944 m water depth, $1.1 \%$ ). Further isotopic work will be required to substantiate if the ${ }^{18} \mathrm{O}$ enrichment at $3.2 \mathrm{Ma}$ is indeed depth dependent.

\section{BENTHIC CARBON ISOTOPIC RECORDS}

The $\delta^{13} \mathrm{C}$ distribution in the Atlantic provides a valuable fingerprint for distinguishing water masses from each other. In most cases, the Atlantic $\delta^{13} \mathrm{C}$ data parallel the distribution of dissolved $\mathrm{O}_{2}$. For example, AABW can be identified by its low $\delta^{13} \mathrm{C}$ value north to the equator; and the core of NADW, with its $\delta^{13} \mathrm{C}$ maximum, can be traced as far as $40^{\circ} \mathrm{S}$ (Kroopnick, 1980). Near the latitude of the Vema Channel $\left(\sim 30^{\circ} \mathrm{S}\right)$, AABW has a $\delta^{13} \mathrm{C}$ value near $0.5 \%$, NADW has a $\delta^{13} \mathrm{C}$ composition near $1.0 \%$, and CPDW has values near $0.65 \%$ (Table 8 ). Because the differences in carbon isotopic values between water masses are large, it should be possible to detect changes in the vertical water mass structure of the Vema Channel by monitoring changes in bathymetric $\Delta^{13} \mathrm{C}$ gradients.

Unfortunately, changes in the $\delta^{13} \mathrm{C}$ of the oceans through time are poorly understood. The synchroneity of carbon isotopic changes in different ocean basins suggests that the $\delta^{13} \mathrm{C}$ record is at least partly controlled by a global mechanism. Recent theories have included transfer of terrestrial or shelf carbon to the oceans (Shackleton, 1977; Broecker, 1981), changes in the C:P ratio of seawater (Broecker, 1981), changes in organic carbon burial rates (Scholl and Arthur, 1980), and changes in oceanic circulation (Bender and Keigwin, 1979). In this study, we are most interested in identifying those changes in the benthic $\delta^{13} \mathrm{C}$ record that are related to changes in local deep circulation. In a similar study, Curry and Lohmann (1982) assumed that global changes in $\delta^{13} \mathrm{C}$ will equally affect all water masses within the mixing time of the oceans. Thus, changes in the bathymetric distribution of $\delta^{13} \mathrm{C}$ should be related to changes in abyssal circulation.

Benthic carbon isotopic records have been generated for the Pliocene of Hole 516A (Leonard et al., this volume), the late Pliocene of Site 517, and the Pliocene of Site 518. Carbon isotopic gradients $\left(\Delta^{13} \mathrm{C}\right)$ have been calculated between sites whenever possible. We assume that the $\Delta^{13} \mathrm{C}$ gradients between Holes 516A $(1313 \mathrm{~m})$, $517(2963 \mathrm{~m})$, and $518(3944 \mathrm{~m})$ reflect the degree of similarity and/or difference in the nutrient content of intermediate, deep, and bottom water masses, respectively. All isotopic analyses were made from specimens of $P$. wuellerstorfi and/or $C$. kullenbergi. $P$. wuellerstorfi accurately reflects the isotopic gradient $(\sim 1 \%)$ between Atlantic and Pacific oceans (Belanger et al., 1981; Graham et al., 1981). In the Vema Channel, Curry and Lohmann (1982) and Williams and Healy-Williams (1982) have shown that the $\delta^{13} \mathrm{C}$ of $P$. wuellerstorfi correctly records the $\triangle^{13} \mathrm{C}$ gradient between $\mathrm{AABW}$ and NADW.

The benthic carbon isotopic record at Site 518 can be divided into three segments (Fig. 8). The interval between 4.1 and $3.6 \mathrm{Ma}$ is marked by relatively light $\delta^{13} \mathrm{C}$ values. At $3.6 \mathrm{Ma}$, a $0.5 \%$ peak $(0.26 \%$ average $)$ enrichment occurs, and heavy values persist till 3.3 Ma. At 3.3 $\mathrm{Ma}, \delta^{13} \mathrm{C}$ values begin a lightening trend culminating in a $1.0 \% 0(0.49 \%$ average $)$ depletion.

The $\Delta^{13} \mathrm{C}$ gradient between Holes $516 \mathrm{~A}$ and 518 is completely dominated by $\delta^{13} \mathrm{C}$ changes in the deeper Site 518 (Fig. 9). This suggests that fundamental circulation 


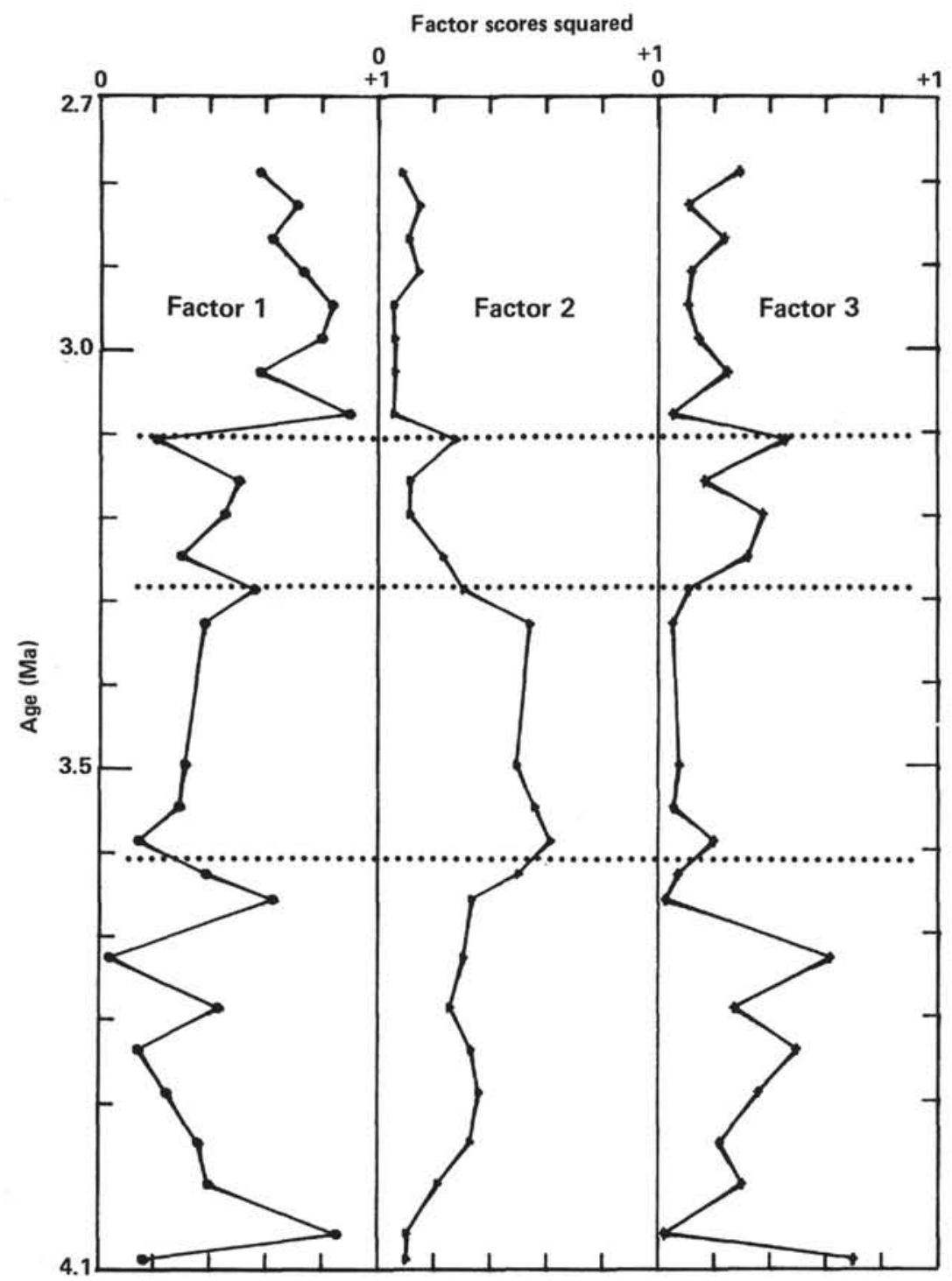

Figure 4. Relative importance of the three most significant benthic foraminiferal factors versus age in Site 518. Rotated varimax factors have been squared and plotted versus age in millions of years. Factor $1=$ Nuttalides umbonifera assemblage (Antarctic Bottom Water); Factor 2 = Globocassidulina subglobosa-Uvigerina peregrina assemblage (Circumpolar Deep Water); Factor $3=$ Oridorsalis umbonatus-Epistominella exigua assemblage (North Atlantic Deep Water). Major changes in the relative importance of benthic foraminiferal assemblages (indicated by dotted lines) occur at 3.6 $\mathrm{Ma}, 3.3 \mathrm{Ma}$, and $3.1 \mathrm{Ma}$. The time scale was derived by isotopic and biostratigraphic correlation with Hole 516A.

changes are occurring within the deeper water masses while the shallower water masses are less affected. The modern gradient between these two depths is -0.15 , reflecting $\delta^{13} \mathrm{C}$ differences between AABW and CPDW. The Pliocene gradient undergoes rapid oscillations during the interval from 4.1 to $3.6 \mathrm{Ma}$, though no permanent change is observed. From 3.6 to $3.2 \mathrm{Ma}$, the gradient is such that $\delta^{13} \mathrm{C}$ values at Site $518(3944 \mathrm{~m})$ are slightly heavier than those at Hole 516A (1313 m). A large negative increase in the $\Delta^{13} \mathrm{C}$ gradient occurs at 3.2 Ma when Site 518 values lighten by $1.0 \%$ relative to values at Hole $516 \mathrm{~A}$.
The modern $\Delta^{13} \mathrm{C}$ gradient between Sites 518 and 517 is -0.5 reflecting the difference between $\mathrm{AABW}$ and NADW. The $\Delta^{13} \mathrm{C}$ gradient between these sites (Fig. 10) during the late Pliocene (3.3 to $2.8 \mathrm{Ma}$ ) also averages about -0.5 , indicating that the AABW/NADW transition during this interval was similar to its present-day position.

Lastly, the $\Delta^{13} \mathrm{C}$ gradient between Sites $517(2963 \mathrm{~m})$ and $516 \mathrm{~A}(1313 \mathrm{~m})$ averages +0.10 between 3.3 and $2.8 \mathrm{Ma}$ (Fig. 11) compared with the modern gradient of +0.35 , reflecting the difference between NADW and CPDW. The direction of the gradient during the late 


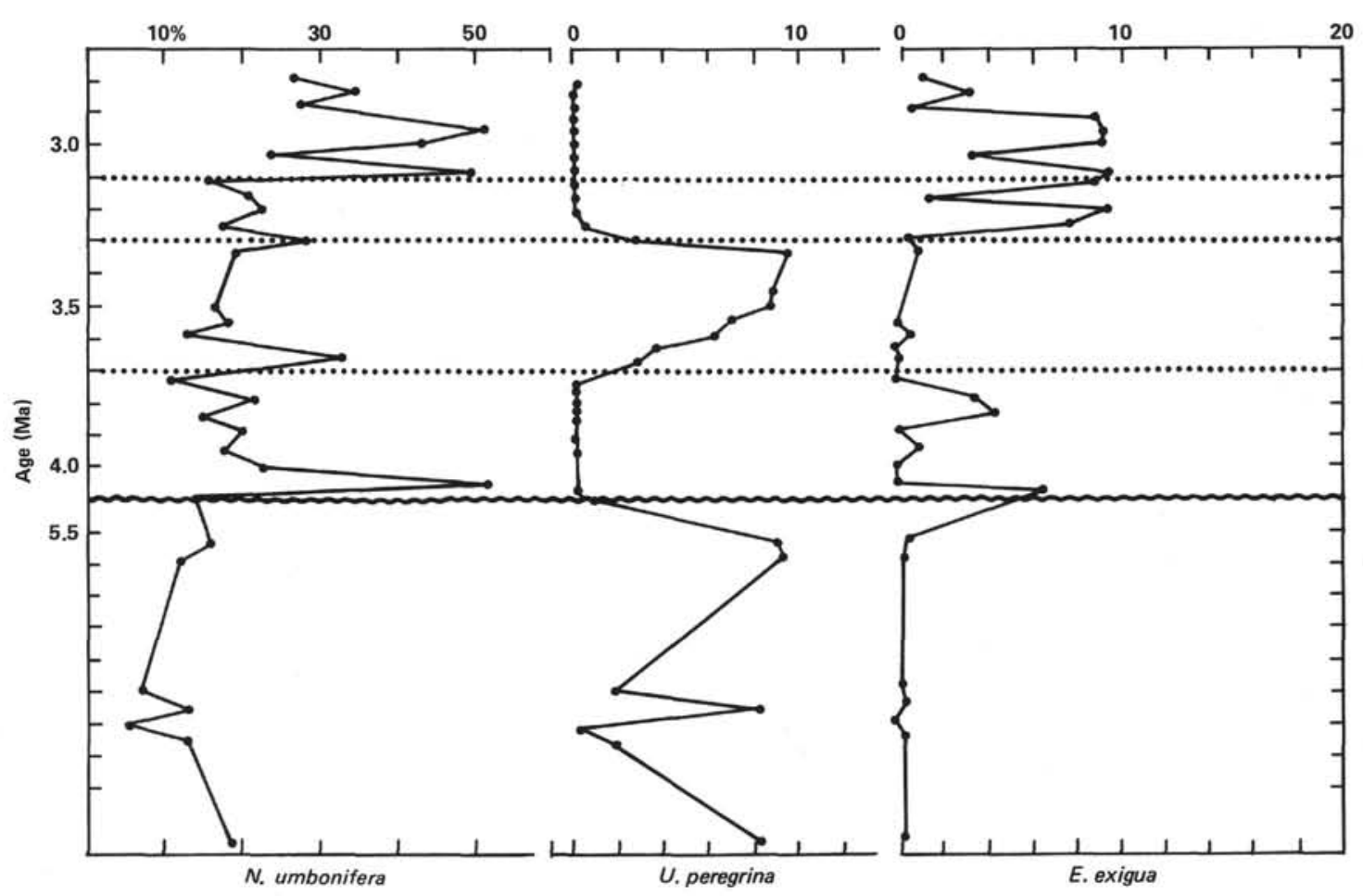

Figure 5. Percentages of Nuttalides umbonifera, Uvigerina peregrina, and Epistominella exigua versus age in Site 518. Wavy line represents a 1.5 Ma hiatus ( 5.5 to $4.0 \mathrm{Ma})$ separating the Miocene and Pliocene in Site 518 . Dotted lines indicate times of major faunal turnovers which are coincident with major changes in the benthic stable isotopic record.

Pliocene is correct though the magnitude is slightly different from the modern gradient.

\section{DISCUSSION}

Changes in the benthic foraminiferal assemblages at Site 518 are coincident with bathymetric carbon isotopic changes, indicating a consistent response to circulation changes. The Pliocene record can be divided into three segments.

The interval between 4.1 and $3.6 \mathrm{Ma}$ is marked by a dominance of the $N$. umbonifera (AABW) and $O$. umbonatus-E. exigua (NADW) assemblages with relatively light $\delta^{13} \mathrm{C}$ values in Hole $518(3944 \mathrm{~m})$. The $\delta^{13} \mathrm{C}$ gradient between Holes $518(3944 \mathrm{~m})$ and 516A (1313 m) undergoes rapid oscillations though no permanent change in the gradient is recorded. The vertical water mass structure of the Vema Channel during this interval was probably similar to the present, with both AABW and NADW present in the western South Atlantic. A major cooling trend was initiated in the southern oceans at 4.3 Ma (Ciesielski and Weaver, 1974). Evidence for this cooling trend includes a northward displacement of siliceous sediments off East Antarctica at $3.86 \mathrm{Ma}$ (Weaver, 1973), possible expansion of the west Antarctic ice sheet to its present dimensions as early as 3.95 to $3.90 \mathrm{Ma}$ (Ciesielski and Weaver, 1974), and major glaciation in Argentine Patagonia at $3.59 \mathrm{Ma}$ (Mercer, 1976). Osborn and others (1982) have found a hiatus maximum in the southwest Indian Ocean which suggests increased bottom water activity in response to the re-establishment of the west Antarctic ice sheet in the late Gilbert Chron $(\sim 4.2$ to $3.6 \mathrm{Ma})$.
From 3.6 to $3.2 \mathrm{Ma}$, the $N$. umbonifera (AABW) and $O$. umbonatus-E. exigua (NADW) assemblages are replaced by a $G$. subglobosa-U. peregrina (CPDW) assemblage, $\delta^{13} \mathrm{C}$ values lighten by $0.5 \%$ in Site 518 , and no strong $\Delta^{13} \mathrm{C}$ gradient exists between Holes 518 $(3944 \mathrm{~m})$ and $516 \mathrm{~A}(1313 \mathrm{~m})$. The presence of a faunal assemblage indicative of CPDW at $3944 \mathrm{~m}$, coupled with the absence of a strong $\Delta^{13} \mathrm{C}$ gradient between $3944 \mathrm{~m}$ (Site 518) and $1313 \mathrm{~m}$ (Hole 516A) suggests that shallow circumpolar waters expanded to depths of at least $3944 \mathrm{~m}$ during this interval. This circulation change may be related to diminished production of AABW and NADW in response to the formation of the Northern Hemisphere Barents Ice Sheet at $\sim 3.5 \mathrm{Ma}$ (Osborn et al., 1982).

The most profound shift in all parameters at Site 518 occurs between 3.3 and $3.2 \mathrm{Ma}$. The G. subglobosa-U. peregrina (CPDW) assemblage is rapidly replaced by the $N$. umbonifera (AABW) and O. umbonatus-E. exigua (NADW) assemblages, the $\Delta^{13} \mathrm{C}$ gradient between Holes 518 and 516A records a large negative increase, and a $1.1 \%$ enrichment is recorded in benthic $\delta^{18} \mathrm{O}$. These changes record the upward displacement by AABW and NADW of shallow circumpolar waters, which had expanded to depths of $3944 \mathrm{~m}$ prior to $3.2 \mathrm{Ma}$. This change in vertical water mass structure was probably related to a conspicuous global cooling event at $3.2 \mathrm{Ma}$ (Kennett et al., 1979; Thunell, 1979) and/or the final closure of the Central American Seaway by 3.0 Ma (Keigwin, 1982).

In order to reconstruct the vertical water mass structure of the Vema Channel during this late Pliocene interval, we have averaged $\delta^{13} \mathrm{C}$ values for each site between 


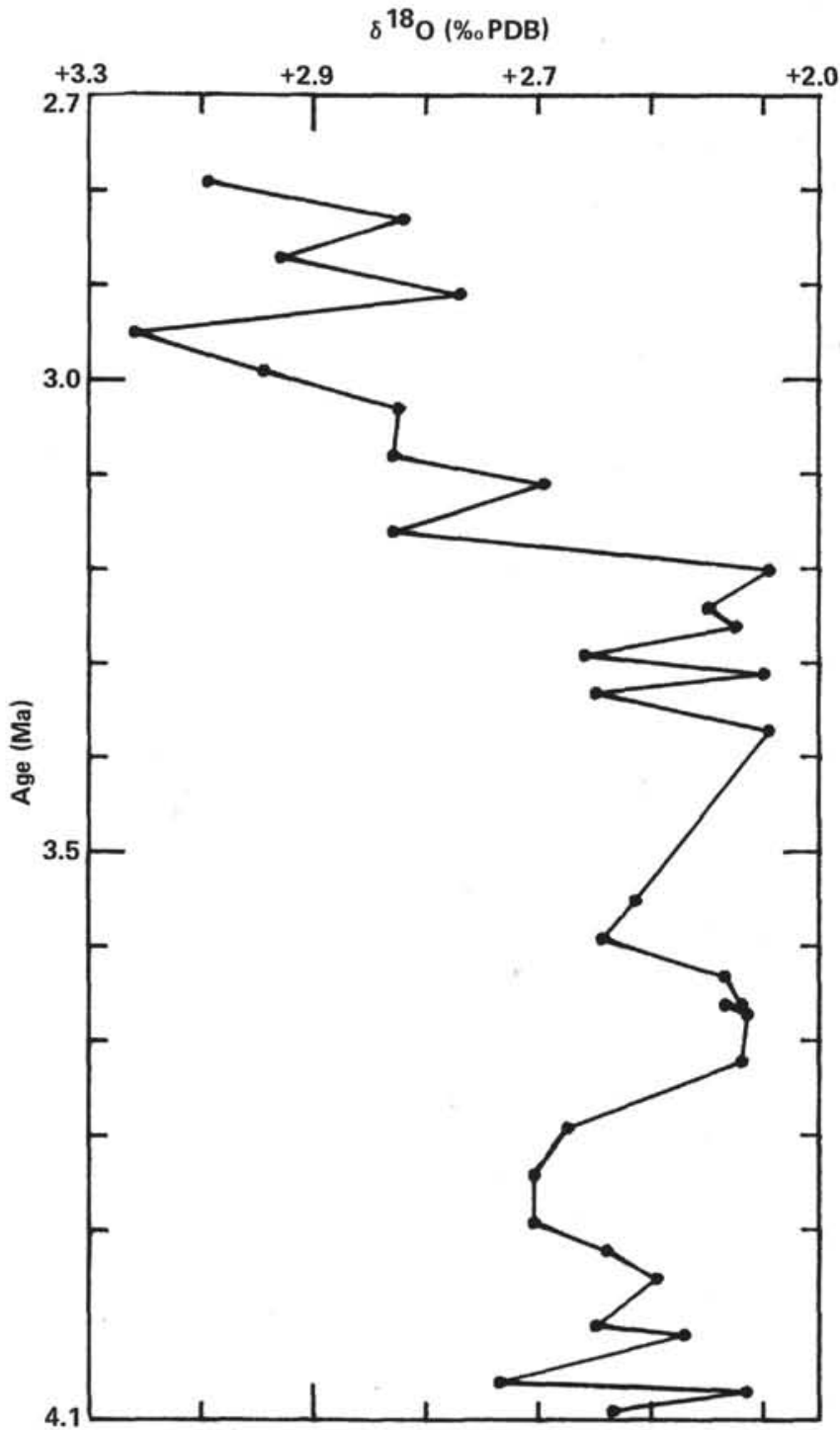

Figure 6. Benthic $\delta^{18} \mathrm{O}(\%$ PDB) from analyses of Planulina wuellerstorf $i$ and/or Cibicidoides kullenbergi in DSDP Site 518 plotted against age in millions of years.

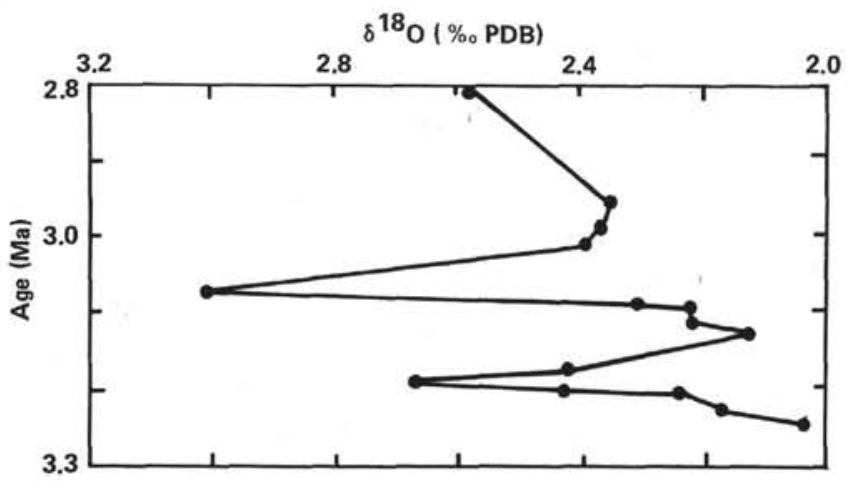

Figure 7. Benthic $\delta^{18} \mathrm{O}(\%$ PDB) from analyses of Planulina wuellerstorfi and/or Cibicidoides kullenbergi in DSDP Site 517 plotted against age in millions of years.
Table 8. Average modern $\delta^{13} \mathrm{C}$ composition (\% PDB) of water masses at the latitude of the Vema Channel in the southwestern Atlantic. $\triangle^{13} \mathrm{C}$ gradients are calculated by taking the $\delta^{13} \mathrm{C}$ difference between adjacent water masses.

\begin{tabular}{ccl}
\hline $\begin{array}{c}\delta^{13} \mathrm{C} \text { (modern) } \\
(\% \text { PDB })^{\mathrm{a}}\end{array}$ & $\begin{array}{c}\Delta^{13} \mathrm{C} \text { gradient } \\
\text { (modern) }\end{array}$ & Water mass $\mathrm{b}$ \\
\hline 1.80 & -0.90 & Surface water \\
0.90 & -0.35 & AAIW \\
0.65 & +0.35 & CPDW \\
1.00 & -0.50 & NADW \\
0.50 & & AABW
\end{tabular}

${ }^{a}$ From Kroopnick, 1980.

b For abbreviations, see Table 1.

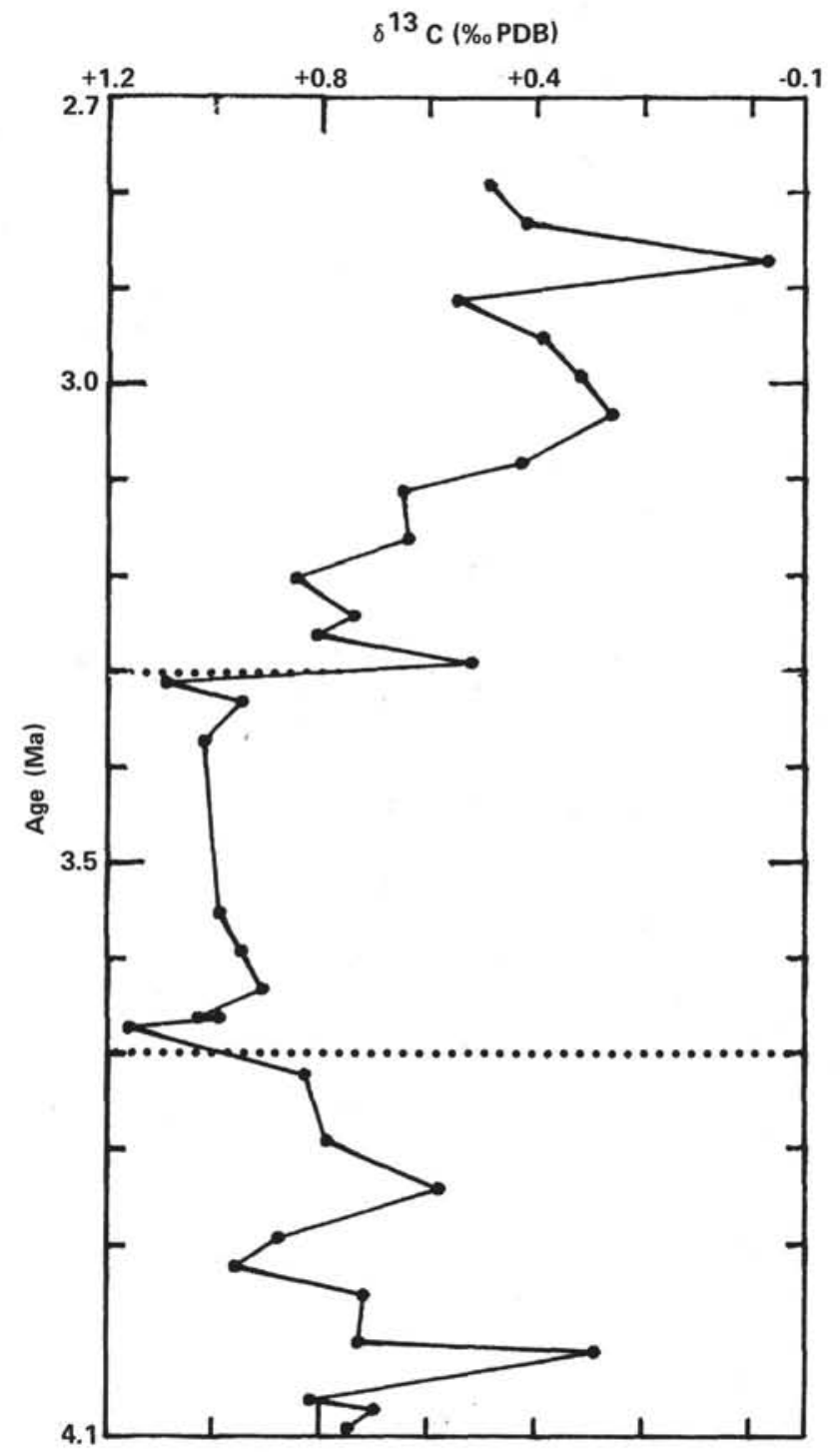

Figure 8. Benthic $\delta^{13} \mathrm{C}(\%, \mathrm{PDB})$ from analyses of Planulina wuellerstorfi and/or Cibicidoides kullenbergi in DSDP Site 518 plotted against age in millions of years. 


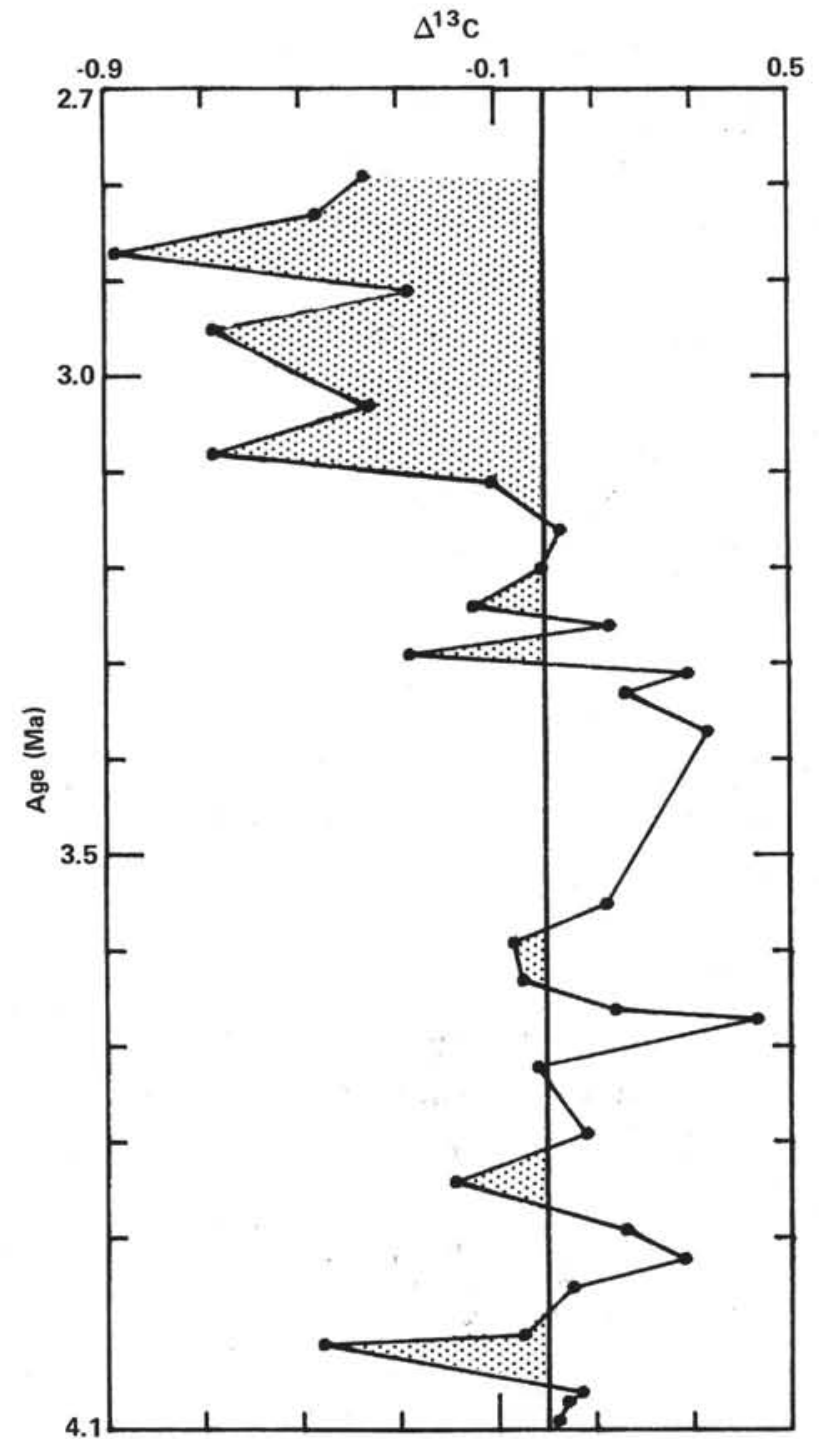

Figure 9. The $\Delta^{13} \mathrm{C}$ gradient between Site $518(3944 \mathrm{~m})$ and Hole 516A $(1313 \mathrm{~m})$ calculated by subtracting the benthic $\delta^{13} \mathrm{C}$ values at Hole $516 \mathrm{~A}$ from values at Site $518 . \Delta^{13} \mathrm{C}$ values are plotted against age in millions of years.

3.2 and $2.7 \mathrm{Ma}$, calculated $\Delta^{13} \mathrm{C}$ differences between sites, and compared the differences with modern $\Delta^{13} \mathrm{C}$ gradients (Table 9). The agreement between the late Pliocene (3.2 to $2.7 \mathrm{Ma}$ ) and present-day gradients indicates that circulation patterns during this late Pliocene interval were similar to those of the modern western South Atlantic.

Ledbetter (1981) suggests that AABW production diminished at $3.2 \mathrm{Ma}$ in the southeastern Indian, the South Atlantic, and the South Pacific oceans. He proposes that decreased AABW activity at $3.2 \mathrm{Ma}$ can be attributed to a decrease in NADW production, which provides part of the salinity component of AABW. Our study does not record circulation intensity, but rather monitors the positions of water masses in the Vema Channel through the Pliocene. Benthic foraminifers and $\Delta^{13} \mathrm{C}$ gradients between 3.2 and $2.7 \mathrm{Ma}$ indicate that both AABW and NADW were present in the Vema

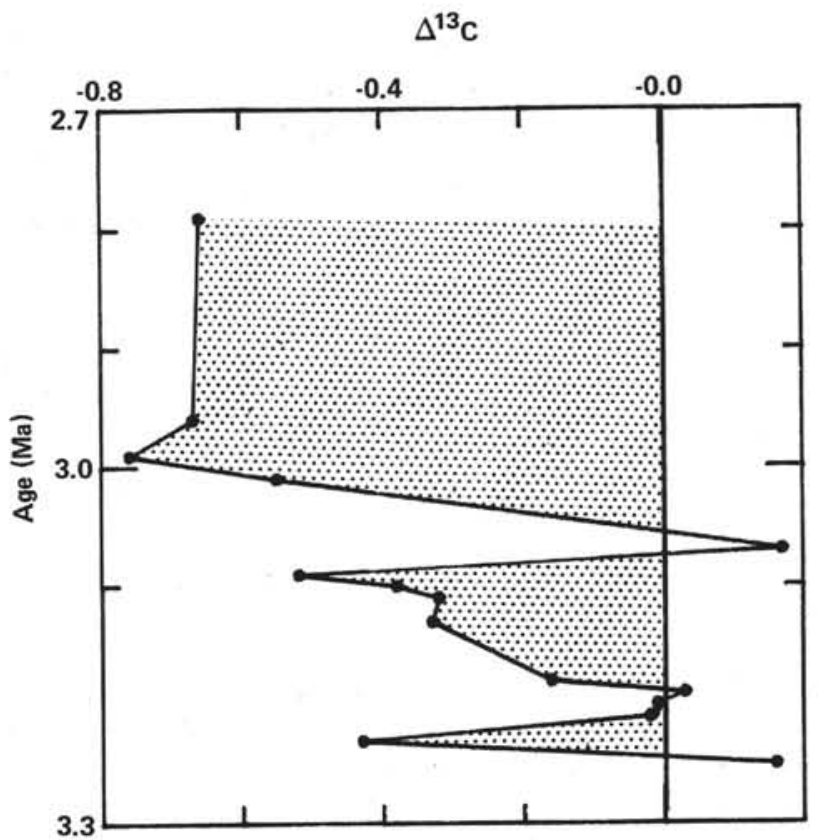

Figure 10. The $\Delta^{13} \mathrm{C}$ gradient between Sites $518(3944 \mathrm{~m})$ and 517 ( $2963 \mathrm{~m}$ ) calculated by subtracting benthic $\delta^{13} \mathrm{C}$ values at Site 517 from values at Site $518 . \Delta^{13} \mathrm{C}$ values are plotted against age in millions of years.

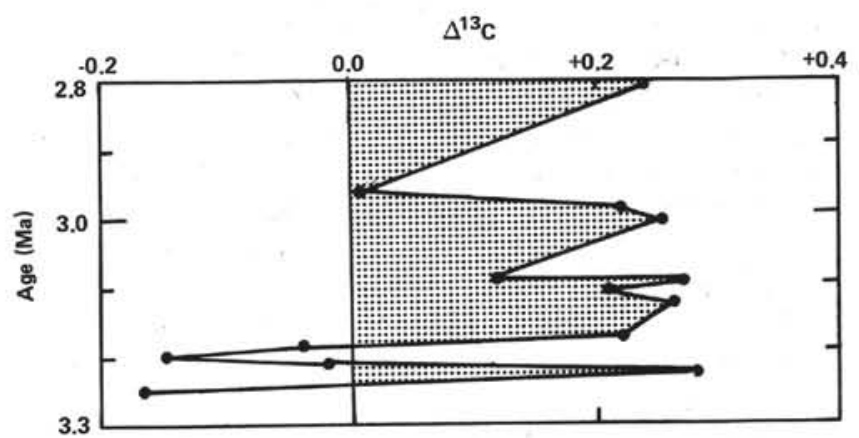

Figure 11. The $\Delta^{13} \mathrm{C}$ gradient between Site $517(2963 \mathrm{~m})$ and Hole 516A $(1313 \mathrm{~m})$ calculated by subtracting benthic $\delta^{13} \mathrm{C}$ values at Hole 516A from values at Site $517 . \Delta^{13} \mathrm{C}$ values are plotted against age in millions of years.

Channel during this interval. The data suggest that the vertical water mass structure of the Vema Channel during the late Pliocene (3.2 to $2.7 \mathrm{Ma}$ ) was similar to the present-day; thus, we do not support decreased production of AABW and NADW during this interval. Keigwin (1982) has found an increased contrast between Atlantic and Pacific benthic foraminiferal $\delta^{13} \mathrm{C}$ at $3.0 \mathrm{Ma}$, which suggests that NADW production actually increased at this time.

\section{CONCLUSIONS}

1. Quantitative analyses of benthic foraminifers from the Pliocene of Site 518 reveal three assemblages which are indicative of specific water masses in the geologic past. These include: an $N$. umbonifera assemblage associated with $\mathrm{AABW}$, a $G$. subglobosa-U. peregrina as- 
Table 9. Averaged $\delta^{13} \mathrm{C}$ values (\% PDB) from planktonic foraminifers and Holes 516A, 517, and 518 benthic foraminifers for the interval between 3.2 and $2.7 \mathrm{Ma} . \Delta^{13} \mathrm{C}$ gradients are calculated between Holes $516 \mathrm{~A}, 517$, and 518 , and compared with modern $\Delta^{13} \mathrm{C}$ radients from Kroopnick (1980). The similarity of modern carbon isotopic gradients with those calculated between 3.2 and 2.7 Ma indicates that the vertical water mass structure of the Vema Channel during the late Pliocene was similar to that of the present day. Sites 516A, 517, and 518 were positioned in CPDW, NADW, and AABW respectively, during the interval from 3.2 to $2.7 \mathrm{Ma}$.

\begin{tabular}{lccccc}
\hline \multirow{2}{*}{$\begin{array}{c}\text { Hole depth } \\
(\mathrm{m})\end{array}$} & $\delta^{13} \mathrm{C}(\% \text { PDB })^{\mathrm{a}}$ & & \multicolumn{2}{c}{$\Delta^{13} \mathrm{C}$ gradient } & \multirow{2}{*}{$\begin{array}{c}\text { Modern } \\
\text { water mass } \\
\text { transition }\end{array}$} \\
\hline Planktonics & $3.2-2.7 \mathrm{Ma}$ & From Kroopnick, 1980 & \\
$516 \mathrm{~m}$ & 1313 & 2.08 & -1.26 & -0.90 & Surface/AAIW \\
517 & 2963 & 0.82 & +0.10 & -1.25 & Surface/CPDW \\
518 & 3944 & 0.92 & -0.47 & -0.35 & NADW/CPDW \\
& 0.45 & & -0.50 & AABW/NADW \\
\hline
\end{tabular}

a Averaged data between 3.2 and $2.7 \mathrm{Ma}$.

b For abbreviations, see Table 1 .

semblage associated with CPDW, and an O. umbonatus-E. exigua assemblage associated with NADW. Changes in benthic foraminiferal assemblages are coincident with changes in the carbon isotopic record, indicating a consistent response to abyssal circulation changes.

2. Changes in the vertical water mass structure of the Vema Channel during the Pliocene appear to be related to climatic events. Three fundamental changes in water mass structure have been identified.

3. From 4.1 to $3.6 \mathrm{Ma}$, the vertical water mass structure was similar to today, with both AABW and NADW present in the western South Atlantic. This result is consistent with hiatus abundance information from the southwest Indian Ocean (Osborn et al., 1982), which suggests increased bottom water activity during this interval, perhaps associated with the re-establishment of the West Antarctic Ice Sheet in the late Gilbert Chron $(\sim 4.2$ to $3.6 \mathrm{Ma})$.

4. From 3.6 to $3.2 \mathrm{Ma}$, shallow circumpolar waters expanded to depths of at least $3944 \mathrm{~m}$. Evidence for the expansion of a shallower water mass to depths of $\sim 4000 \mathrm{~m}$ include the dominance of a CPDW benthic foraminiferal assemblage at Site $518(3944 \mathrm{~m})$ and lack of a $\Delta^{13} \mathrm{C}$ gradient between Holes 516A $(1313 \mathrm{~m})$ and $518(3944 \mathrm{~m})$.

5. At $3.2 \mathrm{Ma}$, circumpolar waters were vertically displaced by AABW and NADW. This change in vertical water mass structure was probably related to climatic deterioration and/or the final closure of the Central American Seaway at approximately 3.2 Ma. A comparison of the present-day vertical water mass structure of the Vema Channel with a reconstruction between 3.2 and $2.7 \mathrm{Ma}$ indicates that circulation patterns during this late Pliocene interval were similar to those of the modern western South Atlantic.

\section{ACKNOWLEDGMENTS}

The authors would like to thank W. B. Curry, D. A. Dunn, D. A. Johnson, G. P., Lohmann, G. A., Mead, and D. F. Williams, for discussions on various aspects of this work; B. H. Corliss for reviewing the manuscript; D. F. Williams for providing Site 516A data and Site 517 samples; G. A. Mead for assistance with taxonomic identifications; and D. R. Muerdter for computer assistance. This research was supported by NSF Grant \#OCE-7914594.

\section{REFERENCES}

Barker, P. F., Carlson, R. L., Johnson, D. A., and Shipboard Scientific Party, 1981. Deep Sea Drilling Project Leg 72: southwest Atlantic paleocirculation and Rio Grande Rise tectonics. Geol. Soc. Am. Bull., 92:294-309.

Belanger, P. E., Curry, W. B., and Matthews, R. K., 1981. Core-top evaluation of the paleo-oceanography utility of benthic foraminiferal isotopic ratios. Palaeogeogr. Palaeoclimatol. Palaeoecol., 33: 205-220.

Bender, M. L., and Keigwin, L. D., 1979. Speculation about the upper Miocene change in abyssal Pacific dissolved bicarbonate $\delta^{13} \mathrm{C}$. Earth Planet. Sci. Lett., 45:383-393.

Bremer, M. L., and Lohmann, G. P., 1982. Evidence for primary control of the distribution of certain Atlantic Ocean benthonic Foraminifera by degree of carbonate saturation. Deep Sea Res., 12: 987-988.

Broecker, W. S., 1981. Glacial to interglacial changes in ocean and atmosphere chemistry. In Berger, A. (Ed.), Climatic Variations and Variability: Facts and Theories: Boston (Reidel), pp. 111-121.

Ciesielski, P. F., and Weaver, F. M., 1974. Early Pliocene temperature changes in the Antarctic Seas. Geology, 2:511-515.

Corliss, B. H., 1978a. Studies of deep-sea benthonic Foraminifera in the Southern Ocean (Ph.D. dissert.). University of Rhode Island. , 1978b. Studies of deep-sea benthonic foraminifera in the southeast Indian Ocean. Antarctic J., 13:116-117. 1979a. Taxonomy of Recent deep-sea benthonic foraminifera from the southeast Indian Ocean. Micropaleo., 25(1):1-19.

1979b. Recent deep-sea benthonic foraminiferal distributions in the southeast Indian Ocean: Inferred bottom-water routes and ecological implications. Mar. Geol., 31:115-138. 1982. Linkage of North Atlantic and Southern Ocean deepwater circulation during glacial intervals. Nature, 298:458-460. 1983. Distribution of Holocene deep-sea benthonic foraminifera in the southwest Indian Ocean. Deep-Sea Res., 30:95-117.

Culp, S. K., 1977. Recent benthic Foraminifera of the Ontong Java Plateau [M.S. Thesis]. University of Hawaii, Honolulu.

Curry, W. B., and Lohmann, G. P., 1982. Carbon isotopic changes in benthonic Foraminifera from the western South Atlantic: Reconstruction of glacial abyssal circulation patterns. Quat. Res., 18: 218-235.

Duplessy, J. C., Moyes, J., and Pujol, C., 1980. Deep water formation in the North Atlantic Ocean during the last ice age. Nature, 286: 479-482.

Gofas, S., 1978. Une approche du paléoenvironment océanique: Les foraminifères benthiques calcaires, traceurs de la circulation abyssale (Ph.D. dissert.). L'Université de Bretagne Occidentale, France.

Graham, D. W., Corliss, B. H., Bender, M. L., and Keigwin. L. D., 1981. Carbon and oxygen isotopic disequilibria of Recent deep-sea benthic foraminifera. Mar. Micropaleo., 6:483-497.

Ingle, J. C., Keller, G., and Kolpack, R. J., 1980. Benthic foraminiferal biofacies, sediments, and water masses of the southern PeruChile Trench area, southeastern Pacific Ocean. Micropaleo., 26: 113-150. 
Keigwin, L. D., 1982. Isotopic paleoceanography of the Caribbean and East Pacific: Role of Panama uplift in Late Neogene time. Science, 217:350-353.

Keigwin, L. D., and Thunell, R. C., 1979. Middle Pliocene climatic change from the western Mediterranean from faunal and isotopic trends. Nature, 282:294-296.

Kennett, J. P., Shackleton, N. J., Margolis, S. V., Goodney, D. E, Dudley, W. C., and Kroopnick, P. M., 1979. Late Cenozoic oxygen and carbon isotopic history and volcanic ash stratigraphy DSDP Site 284, South Pacific. Am. J. Sci., 279:52-69.

Klovan, J. E., and Imbrie, J., 1971. An algorithm and Fortran IV for large scale Q-mode factor analysis. Int. Assoc. Math. Geol. J., 3: 61-78.

Kroopnick, P. M., 1980. The distribution of $\delta^{13} \mathrm{C}$ in the Atlantic Ocean. Earth Planet. Sci. Lett., 49:469-484.

Ledbetter, M. L., 1981. Palaeooceanographic significance of bottomcurrent fluctuations in the Southern Ocean. Nature, 294:554-556.

Lohmann, G. P., 1978. Abyssal benthic Foraminifera as hydrographic indicators in the western South Atlantic. J. Foram. Res., 8(1): 6-34.

1981. Modern benthic foraminiferal biofacies: Rio Grande Rise. EOS, 62(45):903. (Abstract)

Mercer, J. H., 1976. Glacial history of southernmost South America. Quat. Res., 6:125-166.

Osborn, N. I., Ciesielski, P. F., and Ledbetter, M. T., 1982. Disconformities and paleoceanography in the southwest Indian Ocean during the last 5.4 million years. Geol. Soc. Am. Abst. Prog., $14: 652$.

Reid, J. L., Nowlin, W. D., and Patzert, W. C., 1977. On the characteristics and circulation of the Southwestern South Atlantic Ocean. J. Phys. Oceanog., 7:62-91.

Resig, J. M., 1981. Biogeography of benthic Foraminifera of the northern Nazca Plate and adjacent continental margin. In Kulm, L. D., Dymond, J., Dasch, E. J., and Hussong, D. M. (Eds.), Nazca
Plate: Crustal Formation and Andean Convergence, Geol. Soc. Am. Mem., 154:619-644.

Schnitker, D., 1974. West Atlantic abyssal circulation during the past 120,000 years. Nature, 248:385-387.

1980. Quaternary deep-sea benthic foraminifers and bottom water masses. Ann. Rev. Earth Planet. Sci., 8:343-70.

Scholl, P. A, and Arthur, M. A., 1980. Carbon isotopic fluctuations in Cretaceous pelagic limestones: Potential stratigraphic and petroleum exploration tool. AAPG Bull., 64:67-87.

Shackleton, N. J., 1977. Carbon-13 in Uvigerina: Tropical rainforest history and the equatorial Pacific carbonate dissolution cycles. In Andersen, N. R., and Malahoff, A. (Eds.), The Fate of Fossil Fuel $\mathrm{CO}_{2}$ in the Oceans: New York (Plenum Press), pp. 401-427.

Shackleton, N. J., and Opdyke, N. D., 1977. Oxygen isotope and paleomagnetic evidence for early Northern Hemisphere glaciation. Nature, 270:216-219.

Streeter, S. S., 1973. Bottom water and benthonic Foraminifera in the North Atlantic: Glacial-interglacial contrasts. Quat. Res., 3(1): 131-141.

Thunell, R. C., 1979. Pliocene-Pleistocene paleotemperature and paleosalinity history of the Mediterranean Sea: Results from DSDP Sites 125 and 132. Mar. Micropaleo., 4:173.

Weaver, F. M., 1973. Pliocene paleoclimatic and paleoglacial history of East Antarctica recorded in deep-sea piston cores. Florida State Univ., Sedimentological Res. Lab., Contrib. 36.

Williams, D. F., and Healy-Williams, N., 1982. Vertical isotope gradients in Recent benthic Foraminifera and the water column of the Vema Channel. EOS, 63:359. (Abstract)

Woodruff, F., Savin, S. M., and Douglas, D. G., 1981. Miocene stable isotope record: A detailed deep Pacific Ocean study and its paleoclimatic implications. Science, 212:665-668.

Date of Initial Receipt: November 2, 1982 